\title{
A Theoretical Study of Favorskii Reaction Stereochemistry: Lessons in Torquoselectivity
}

\author{
Graham D. Hamblin, Raphael P. Jimenez, and Ted S. Sorensen* \\ Department of Chemistry, University of Calgary, 2500 University Drive, Calgary, \\ Alberta T2N1N4 Canada
}

\section{Supporting Information}

\section{$\underline{\text { Table of Contents }}$}

1. Evaluation of Computational Methods for Conducting this Study

S-1

2. Enolate Dihedral Angle (Cl-C-C-O) Profiles for Chloroenolates

1, 2, 8-11.

3. Solvation Energies for Ground-states and Transition-states for 1, 2, 6-14. S-8

4. Comparison of SCI-PCM and PCM Solvation Methods S-8

5. Overall Reaction Energy Calculations $\quad$ S-9

6. Probe of the CCSD(T) TS Energy Profiles $\quad$ S-9

7. Calculated Transition-state Energies (Solvated) S-13

8. Coordinates, ZPVE and Lowest Frequency for all Structures Obtained S-13 in this study.

\section{Evaluation of Computational Methods for Conducting this Study}

Chloroenolate 6 was used for this work.

Table S1: C-Cl Bond Lengths and $\Delta \mathrm{E}^{\ddagger}$ Values (Inv.)

$\begin{array}{cc}\text { Method } & \begin{array}{c}\text { C-Cl Bond Length } \\ \text { (GS) inv. }(\AA)^{1}\end{array} \\ \text { B3LYP/6-31G* }^{*} & 1.892 \\ \text { B3LYP/6-311++G }^{* *} & 1.876 \\ \text { BLYP/6-311+G* }^{*} & 1.963 \\ \text { MP2/6-31G* } & 1.826 \\ \text { MP2/6-311+G** } & 1.815 \\ \text { MP2/6-311+G* } & 1.819 \\ \text { MP4(s,d,q)/6-31G* } & 1.832 \\ \text { MP4(s,d,q)/6-311++G** } & 1.822\end{array}$

C-Cl Bond Length
(TS) inv. (̊)
2.623
2.661
2.819
2.446
2.479
2.487
2.472
2.507

$\Delta \mathrm{E}^{\ddagger}$ (inv.)
$(\mathrm{kcal} / \mathrm{mol})$
13.14
15.59
14.32
18.87
24.00
23.19
19.77
24.89

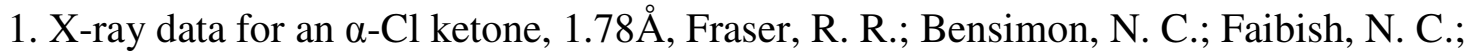
Kong, F., Can. J. Chem., 1994, 72, 1481. See also Laube, T.; Ha, T. K., J. Am. Chem. Soc., 1988, 110, 5511 for a discussion of variation of the $\mathrm{C}-\mathrm{Cl}$ bond lengths with torsion angle. 
2. All calculations were carried out using the Gaussian 03 suite of programs. For the Unrestricted B3LYP calculations, the stability of the wavefunction was tested with the keywords STABLE=OPT and GUESS=MIX. Gaussian 03, Revision A.1, Frisch, M. J.; Trucks, G. W.; Schlegel, H. B.; Scuseria, G. E.; Robb, M. A.; Cheeseman, J. R; Montgomery, Jr., J. A.; Vreven, T.; Kudin, K. N.; Burant, J. C.; Millam, J. M.; Iyengar, S. S.; Tomasi, J.; Barone, V.; Mennucci, B.; Cossi, M.; Scalmani, G.; Rega, N.; Petersson, G. A.; Nakatsuji, H.; Hada, M.; Ehara, M.; Toyota, K.; Tukuda, R.; Hasegawa, J.; Ishida, M.; Nakajima, T.; Honda, Y.; Kitao, O.; Nakai, H.; Klene, M.; Li, X.; Knox, J. E.; Hratchian, H. P.; Cross, J. B.; Adamo, C.; Jaramillo, J.; Gomperts, R.; Stratmann, R. K.; Voth, G. A.; Salvador, P.; Dannenberg, J. J.; Zakrzewski, V. G.; Dapprich, S.; Daniels, A. D.; Strain, M. C.; Farkas, O.; Malick, D. K.; Rabuck, A. D.; Raghavachari, K.; Foresman, J. B.; Ortiz, J. V.; Cui, Q.; Baboul, A. G.; Clifford, S.; Cioslowski, J.; Stefanov, B. B.; Liu, G.; Liashenko, A.; Piskorz, P.; Komaromi, I.; Martin, R. L.; Fox, D. J.; Keith, T.; AlLaham, M. A.; Peng, C. Y.; Nanayakkara, A.; Challacombe, M.; Gill, P. M. W.; Johnson, B.; Chen, W.; Wong, M. W.; Gonzalez, C.; and Pople, J. A.; Guassian, Inc, Pittsburgh PA, 2003.

Table S2: Chloroacetaldehyde C-Cl Bond Lengths

$\begin{array}{ll}\text { B3LYP/6-31G* } & 1.804 \AA \\ \text { MP2/6-31G* } & 1.776 \AA \\ \text { MP4(sdq)/6-31G* } & 1.782 \AA\end{array}$

Table S3: Chloromethane C-Cl Bond Lengths

$\begin{array}{ll}\text { B3LYP/6-31G* } & 1.8027 \AA \\ \text { B3LYP/6-311+G** } & 1.8038 \AA \\ \text { MP2/6-31G* } & 1.7787 \AA \\ \text { MP2/6-311+G** } & 1.7792 \AA \\ \text { MP4(sdq)/6-311+G** } & 1.7899 \AA\end{array}$


Table S4: Further Computational Method Comparisons; Structures $\mathbf{1 0}$ and $\mathbf{1 2}$

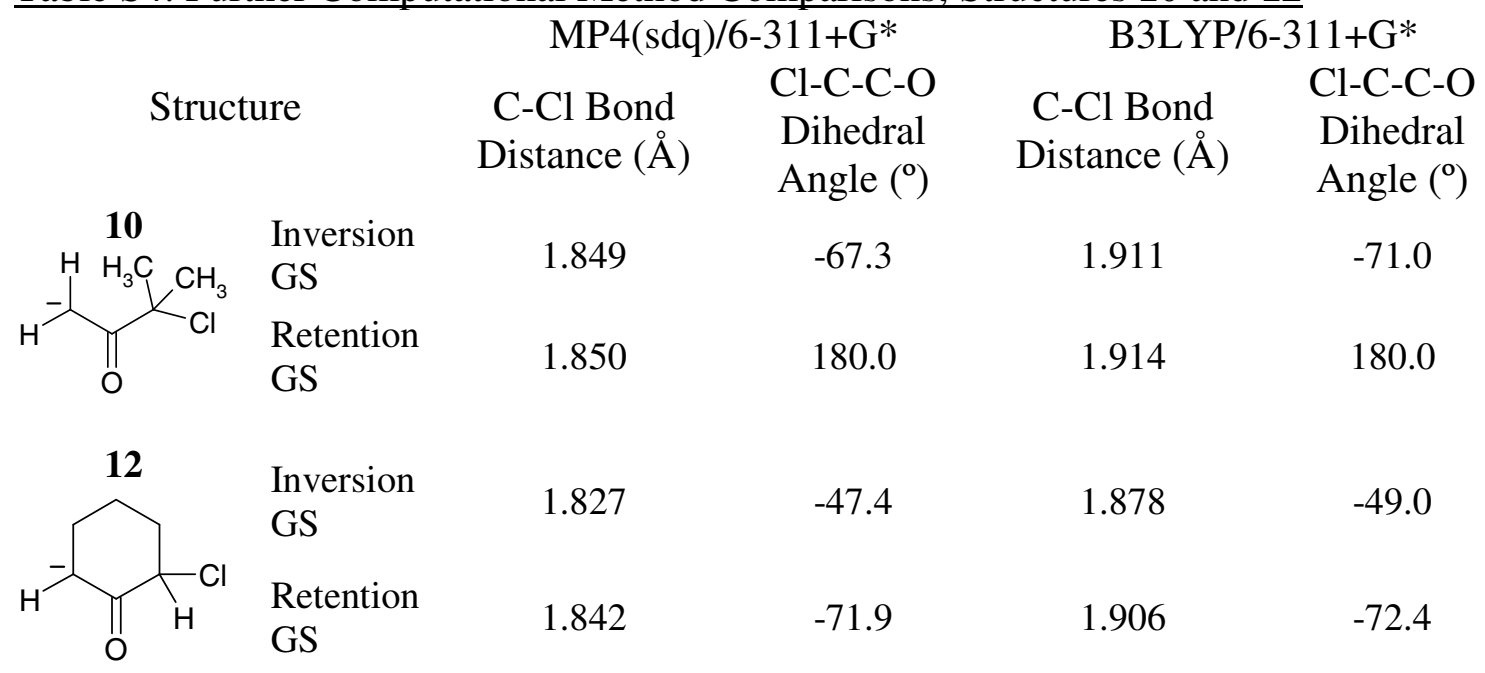

Table S5: Couple Cluster (CCSD(T)) Single Point Energy Calculations of B3LYP, MP2 and MP4/6-311+G* Geometries (Optimized)

$\begin{array}{lc}\text { B3LYP/6-311+G* } & +0.393 \mathrm{kcal} / \mathrm{mol}^{(\mathrm{a})} \\ \text { MP2/6-311+G* } & +0.045 \mathrm{kcal} / \mathrm{mol} \\ \text { MP4(sdq)/6-311+G* } & 0.000 \mathrm{kcal} / \mathrm{mol}\end{array}$

(a) numbers are relative to the lowest energy value $=0.0$

2. Enolate Dihedral Angles (Cl-C-C-O) for Chloroenolates 1, 2, 8-11 


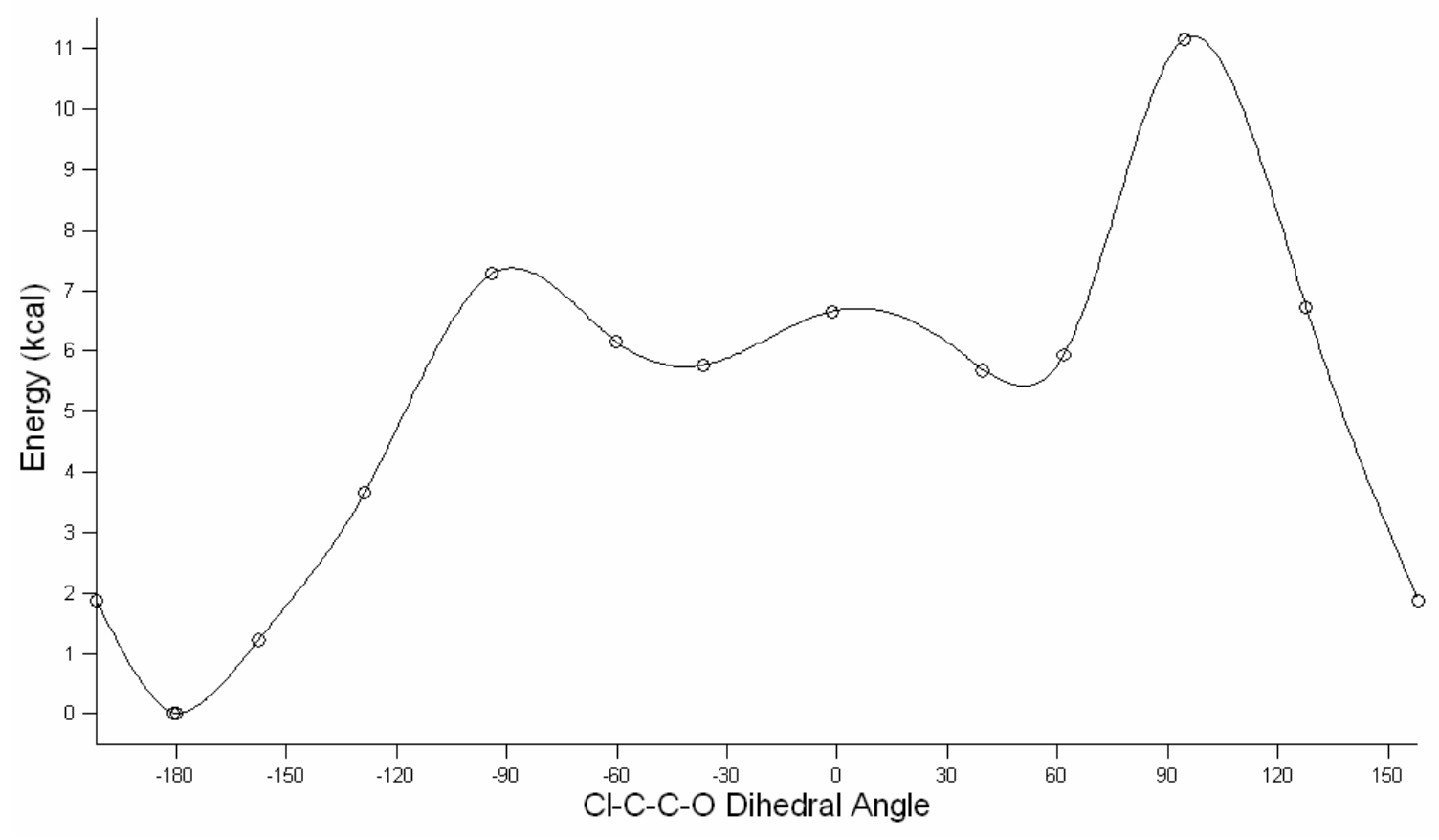

Figure S1: Rotation Profile for Enolate 1 at MP2/6-31G* (Axial Cl conformation)

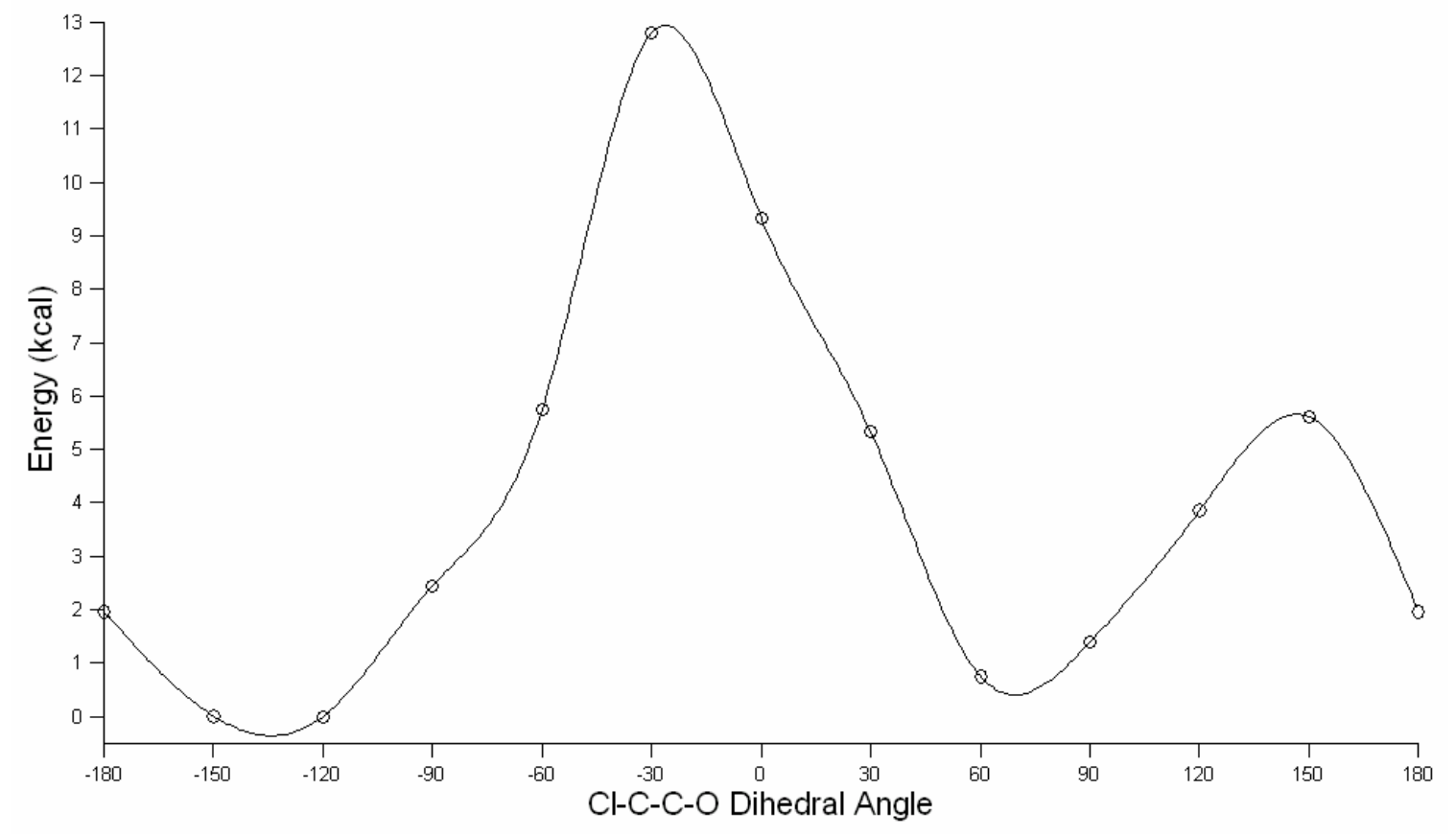

Figure S2: Rotation Profile for Enolate 2 at MP2/6-31G* (Axial Cl conformation) 


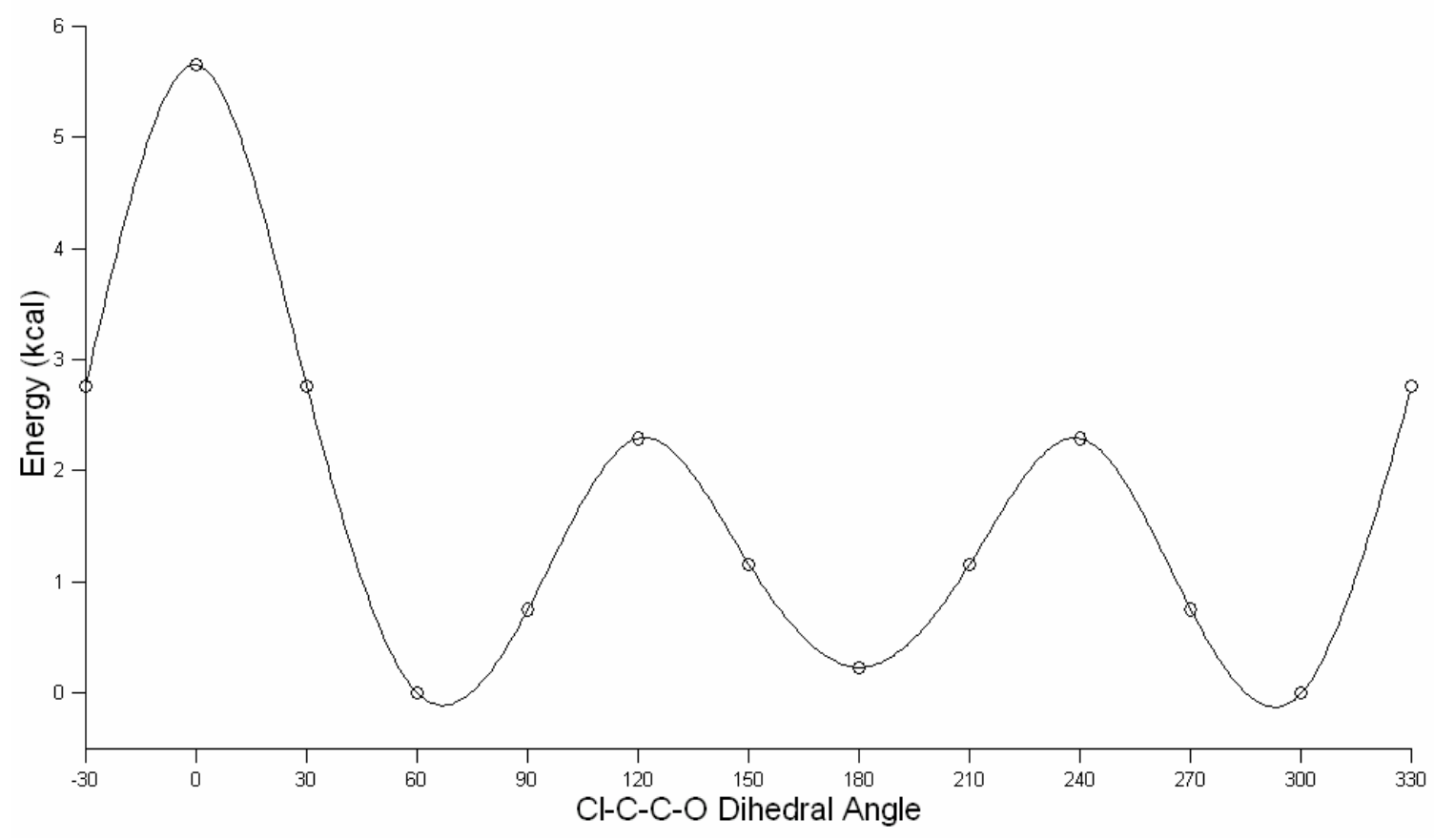

Figure S3: Rotation Profile for Enolate 7 at MP2/6-311+G*

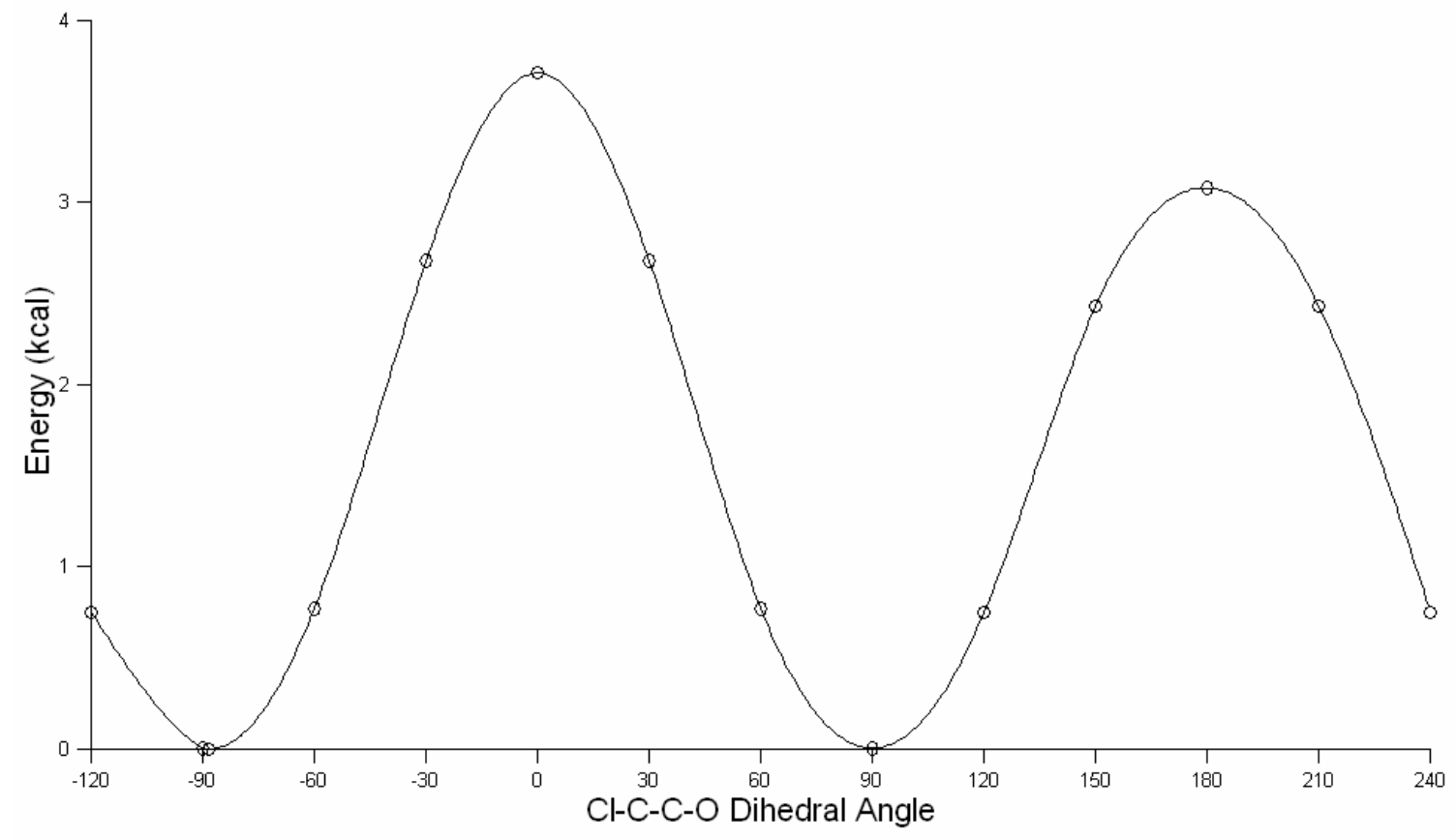

Figure S4: Rotation Profile for Enolate 8 at MP2/6-311+G* 


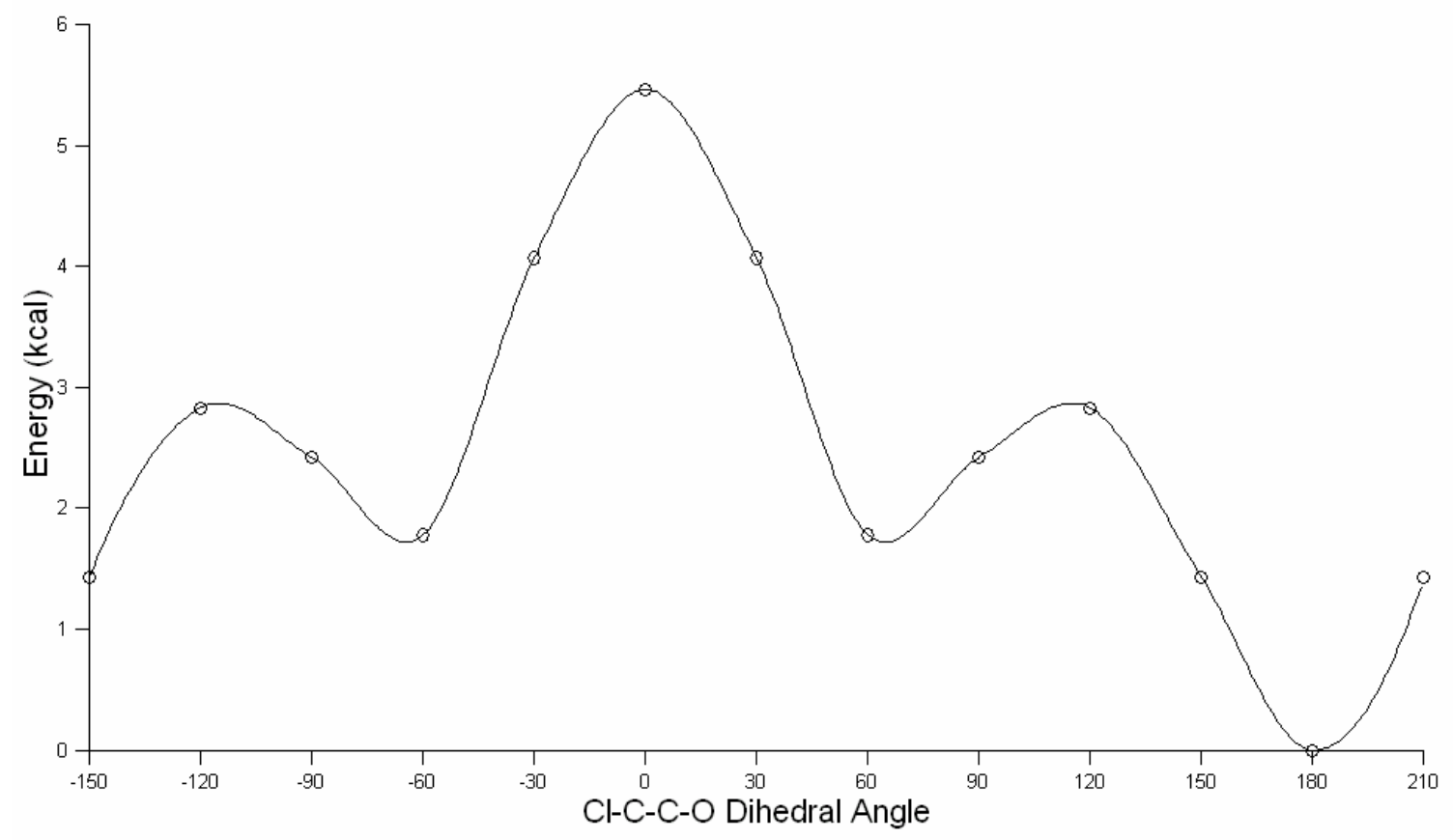

Figure S5: Rotation Profile for Enolate 10 at MP2/6-311+G*

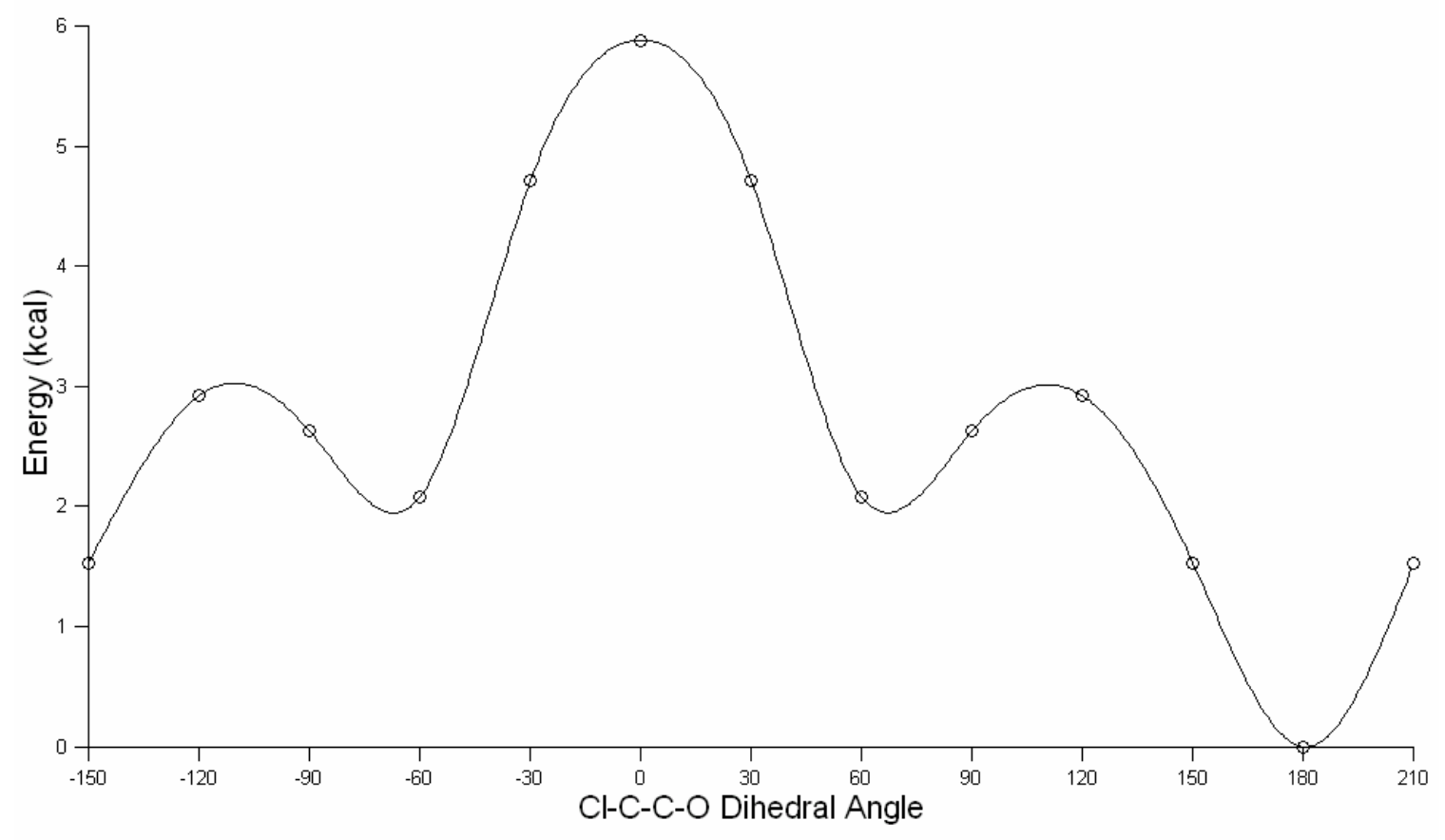

Figure S6: Rotation Profile for Enolate 11 at MP2/6-311+G* 


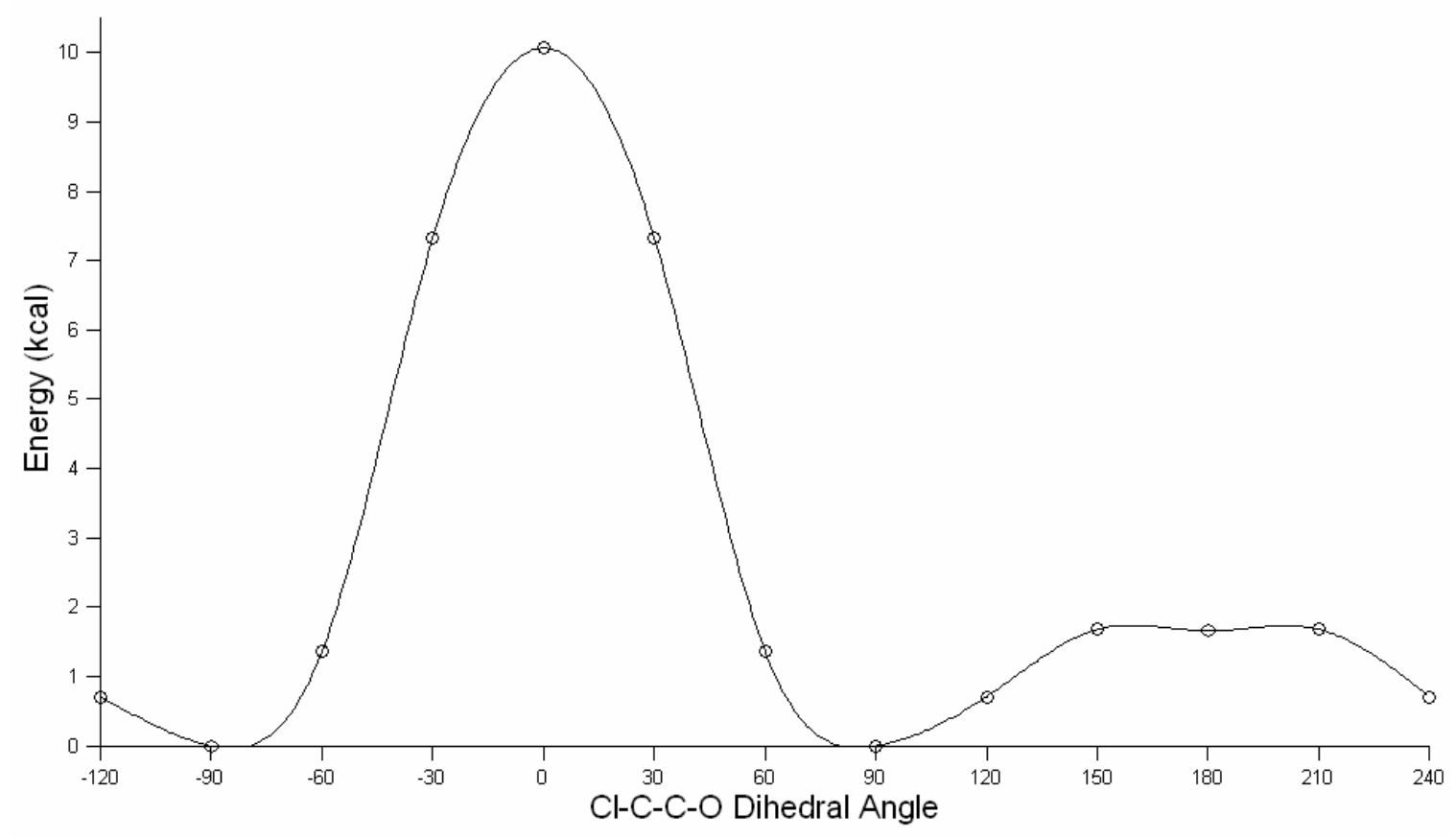

Figure S7: Rotation Profile for Enolate 11 with Equatorial Chlorine at MP2/6-311+G* 


\section{Calculated PCM Solvation Energies for Ground-states and Transition-states for 1, 2, 6-14}

Table S6 Solvation Energies for 1, 2, 6-14 at MP2/6-311+G* with $€=32.6$, methanol

\begin{tabular}{|c|c|c|c|}
\hline Structure & Configuration & $\begin{array}{c}\text { Ground-state Solvation } \\
\text { (Methanol) Energy (kcal/mol) }\end{array}$ & $\begin{array}{c}\text { Transition-state Solvation Energy } \\
\text { (Methanol) }(\mathrm{kcal} / \mathrm{mol})\end{array}$ \\
\hline \multirow{4}{*}{1} & Inversion A & 56.34 & 61.16 \\
\hline & Inversion B & 57.19 & 61.89 \\
\hline & Retention C & 5418 & 59.48 \\
\hline & Retention D & 54.18 & 60.60 \\
\hline \multirow{4}{*}{2} & Inversion E & & 66.35 \\
\hline & Inversion F & 56.54 & 64.17 \\
\hline & Retention G & & 62.54 \\
\hline & Retention H & 54.22 & 62.29 \\
\hline \multirow{2}{*}{6} & Inversion & 59.88 & 63.24 \\
\hline & Retention & 58.35 & 63.47 \\
\hline \multirow{2}{*}{7} & Inversion & 58.48 & 64.86 \\
\hline & Retention & 56.78 & 64.57 \\
\hline \multirow{2}{*}{8} & Inversion & & 63.98 \\
\hline & Retention & 60.28 & 62.71 \\
\hline \multirow{4}{*}{9} & Inversion A & 5844 & 63.67 \\
\hline & Inversion B & 58.44 & 64.20 \\
\hline & Retention C & 5736 & 63.88 \\
\hline & Retention D & $5 \%$ & 63.91 \\
\hline \multirow{2}{*}{10} & Inversion & 58.05 & 64.47 \\
\hline & Retention & 55.57 & 63.59 \\
\hline \multirow{2}{*}{11} & Inversion & 59.05 & 64.46 \\
\hline & Retention & 55.50 & 62.65 \\
\hline \multirow{2}{*}{12} & Inversion & 60.06 & 64.30 \\
\hline & Retention & 60.65 & 66.25 \\
\hline \multirow{2}{*}{13} & Inversion & 59.96 & 64.56 \\
\hline & Retention & 59.92 & 66.70 \\
\hline \multirow{2}{*}{14} & Inversion & 59.44 & 63.95 \\
\hline & Retention & 60.38 & 65.95 \\
\hline
\end{tabular}

\section{Comparison of SCI-PCM and PCM Solvation Methods}

\section{Table S7: SCI-PCM and PCM Solvation Comparison}

$\begin{array}{cccc}\text { Structure } & \text { Gas Phase } & \text { Solvated with } \mathbf{C = 2 5} & \begin{array}{c}\text { Solvated with } \mathbf{C = 2 5} \text { and } \\ \text { Optimized }\end{array} \\ & -652.212521 & -652.312301 & -652.316922 \\ \text { Retention Transition State } & -652.222908 & -652.323106 & -652.326275 \\ \text { Inversion Transition State } & 0.010387 & 0.010805 & 0.009353 \\ \text { Difference Favoring Inversion } & (6.52 \mathrm{kcal} / \mathrm{mol}) & (6.78 \mathrm{kcal} / \mathrm{mol}) & (5.87 \mathrm{kcal} / \mathrm{mol}) \\ \text { SCI-PCM Solvation Minus } & & 6.78-5.87=0.91 \mathrm{kcal} / \mathrm{mol} \\ \text { PCM Solvation } & & & \end{array}$




\section{Reaction Energy Calculations}

Enolate $\rightarrow$ The Cyclopropanone $+\mathrm{Cl}^{-}$

Table S8: Reaction Energy Calculations (MP2/6-311+G*) with Solvation (PCM, C=32.6, $\underline{\text { methanol) }}$

Enolate 6 (inv. GS) Gas-Phase $-11.30 \mathrm{kcal} / \mathrm{mol}^{1}$

Solvated $\quad+7.11 \mathrm{kcal} / \mathrm{mol}^{2}$

Enolate 11 (inv. GS) Gas-Phase $-15.03 \mathrm{kcal} / \mathrm{mol}$

Solvated $\quad+5.93 \mathrm{kcal} / \mathrm{mol}$

Enolate 12 (ret. conf.) Gas-Phase $-13.54 \mathrm{kcal} / \mathrm{mol}$

Solvated $\quad+2.75 \mathrm{kcal} / \mathrm{mol}$

1. Negative sign represents an endothermic process

2. Free energy values for the reaction will be even larger due to a favorable entropy term.

\section{Probe of the CCSD(T) TS Energy Profiles}

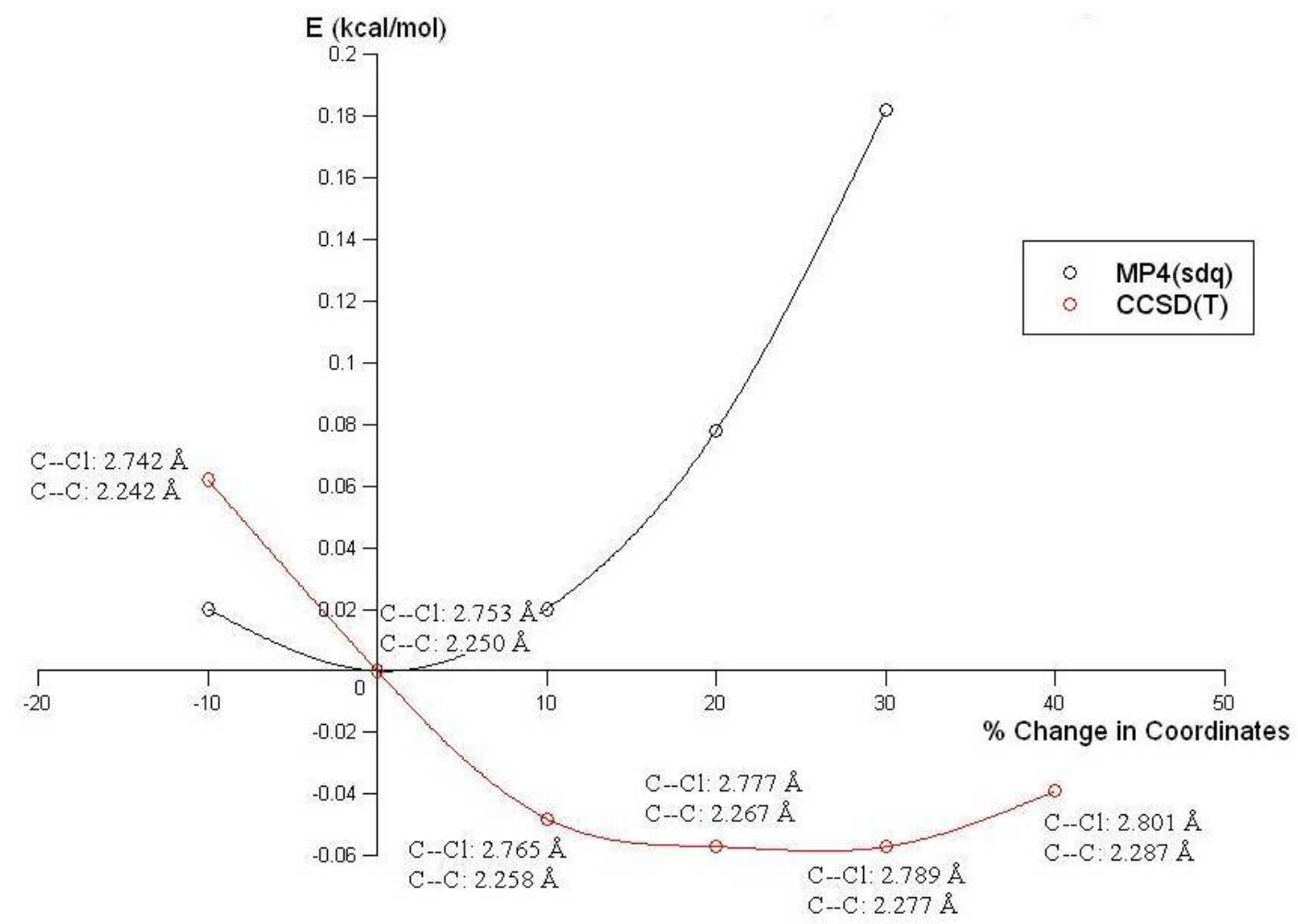

Figure S8. Structure 6 Retention Process at 6-311+G* Method Comparison on Lowest Positive Frequency $\left(91.70 \mathrm{~cm}^{-1}\right)$ coordinates. 


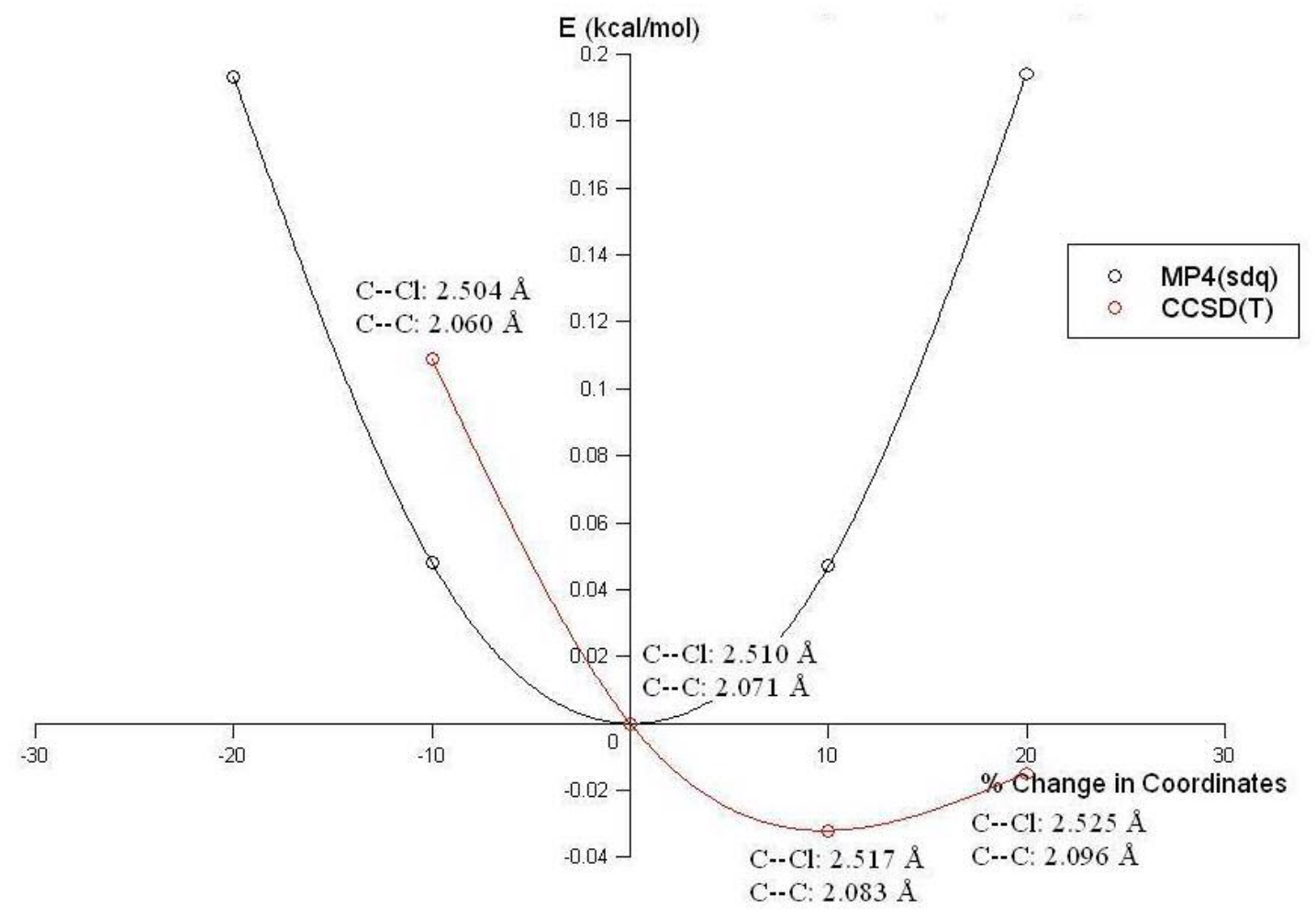

Figure S9. Structure 6 Inversion Process at 6-311+G* Method Comparison on Lowest Positive Frequency $\left(113.25 \mathrm{~cm}^{-1}\right)$ coordinates.

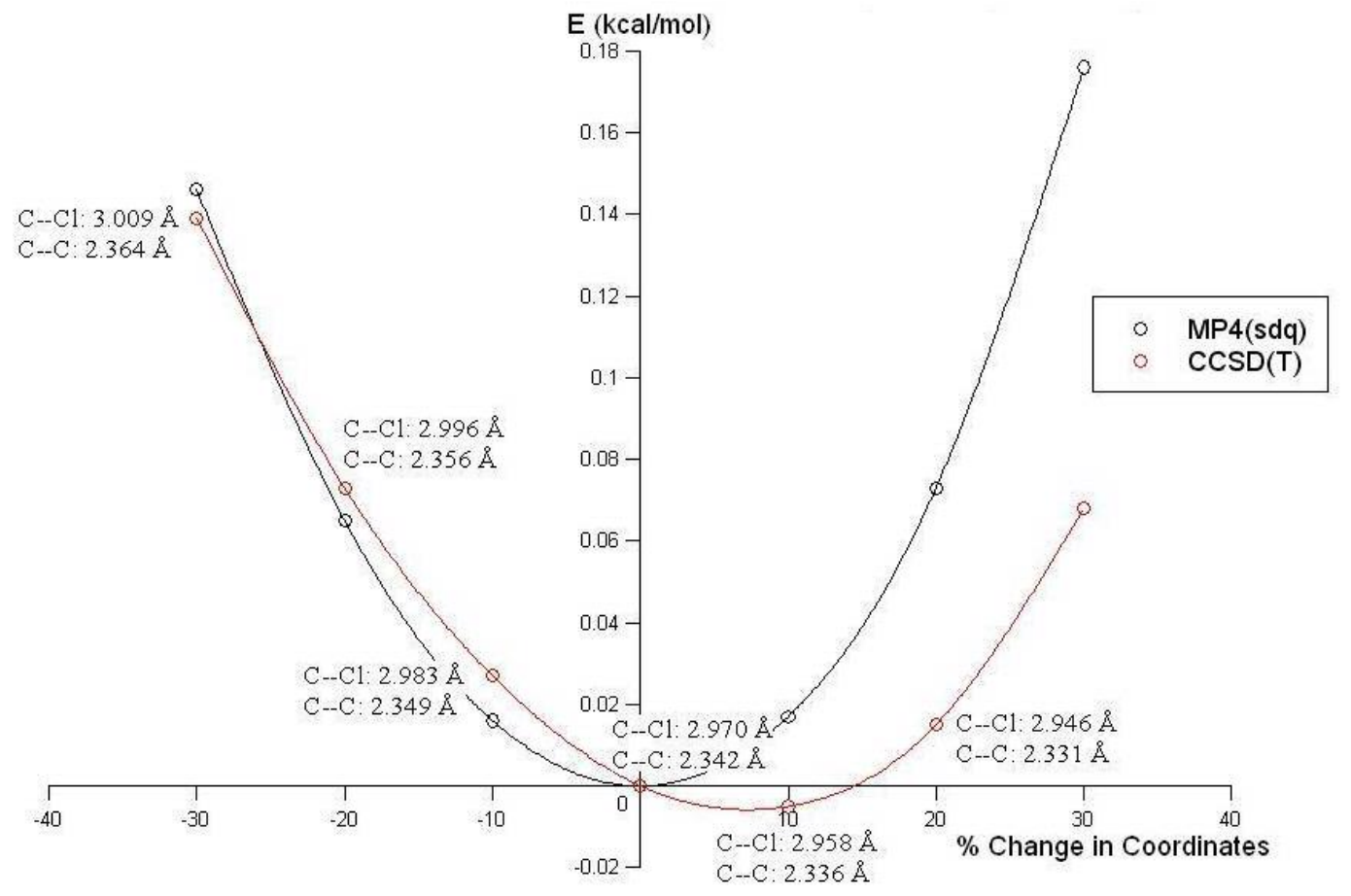

Figure S10. Structure 10 Retention Process at 6-311+G* Method Comparison on $2^{\text {nd }}$ Lowest Positive Frequency $\left(77.49 \mathrm{~cm}^{-1}\right)$ coordinates. 


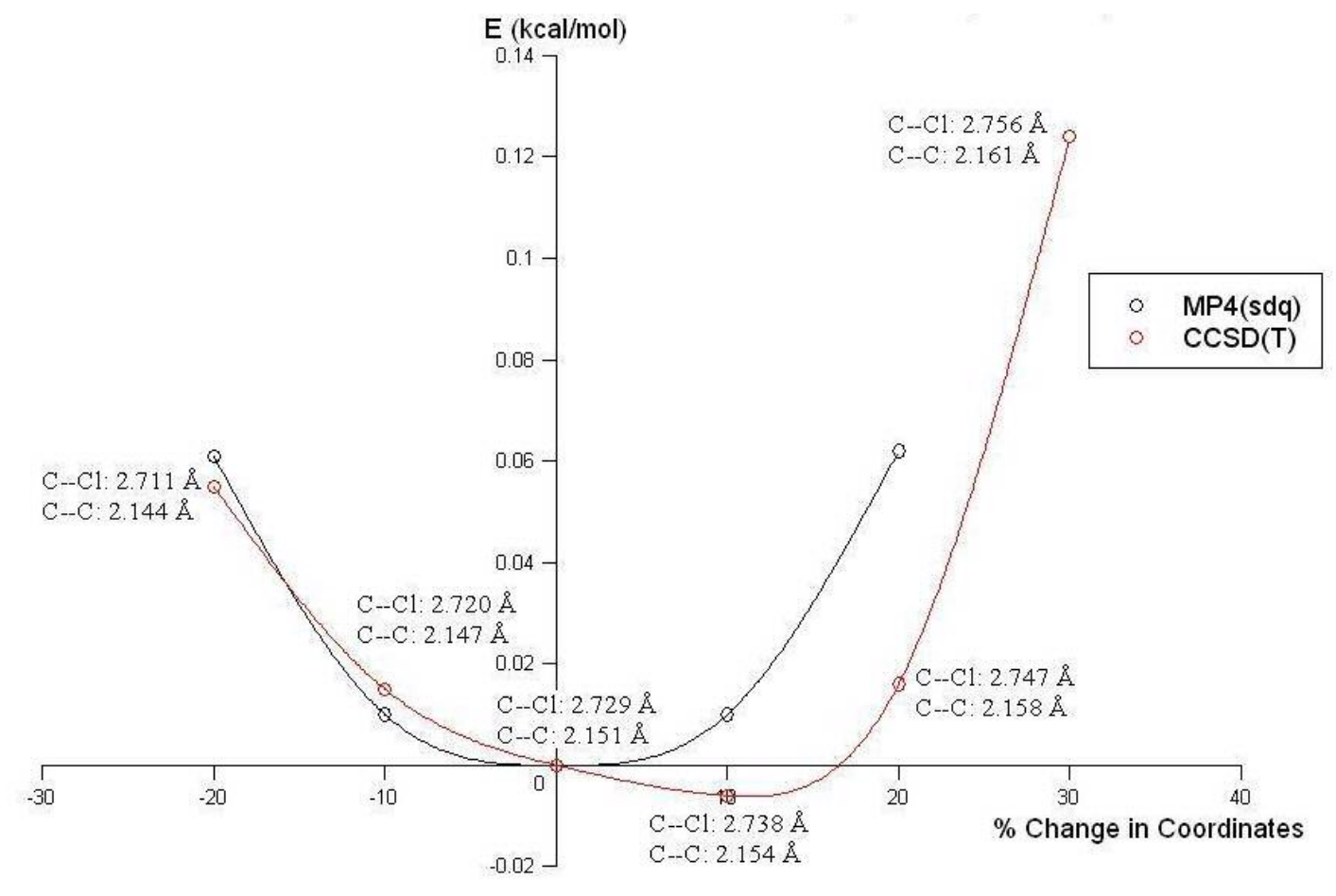

Figure S11. Structure 10 Inversion Process at 6-311+G* Method Comparison on Lowest Positive Frequency $\left(80.75 \mathrm{~cm}^{-1}\right)$ coordinates.

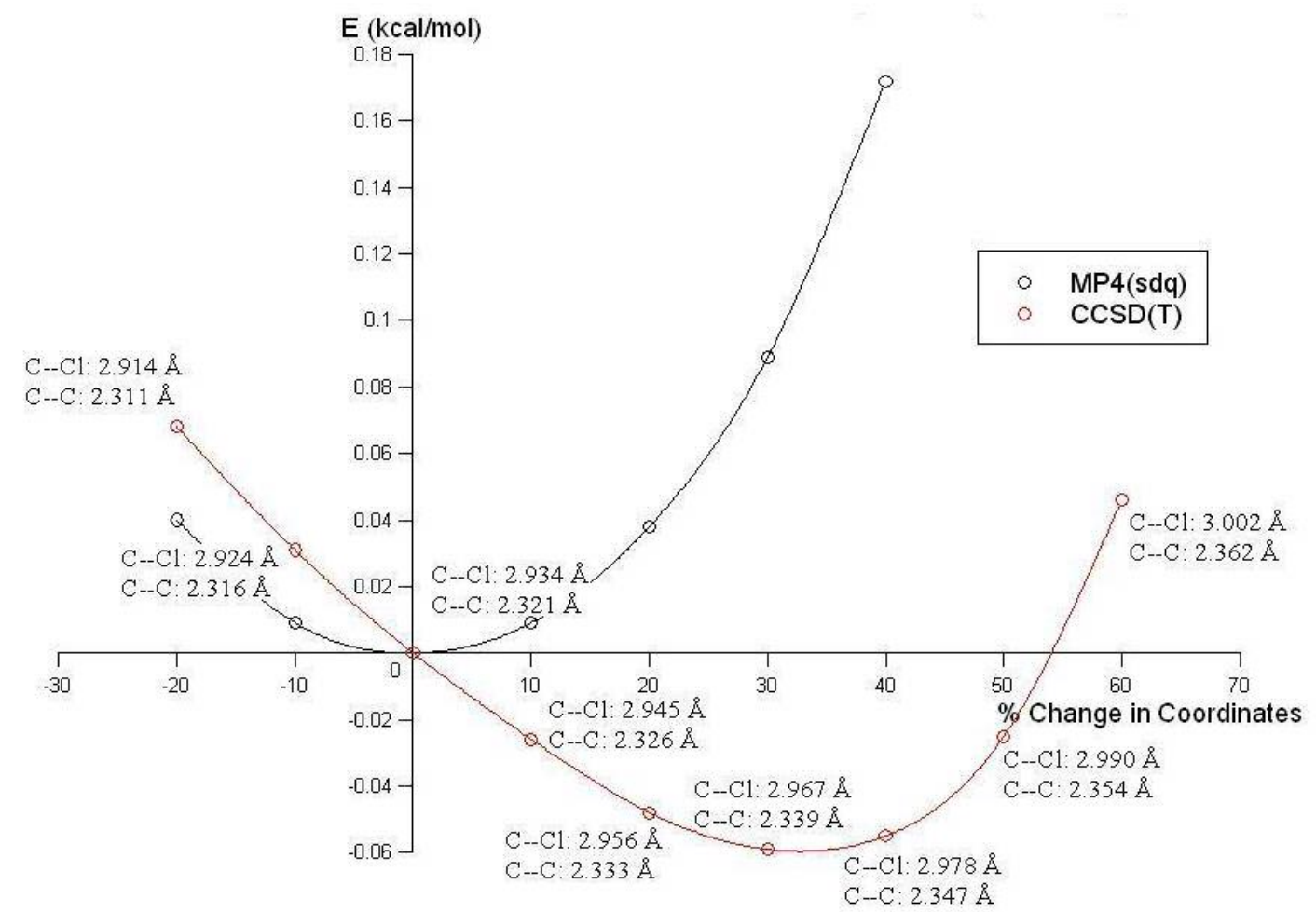

Figure S12. Structure 12 Retention Process at 6-311+G* Method Comparison on Lowest Positive Frequency $\left(60.25 \mathrm{~cm}^{-1}\right)$ coordinates. 


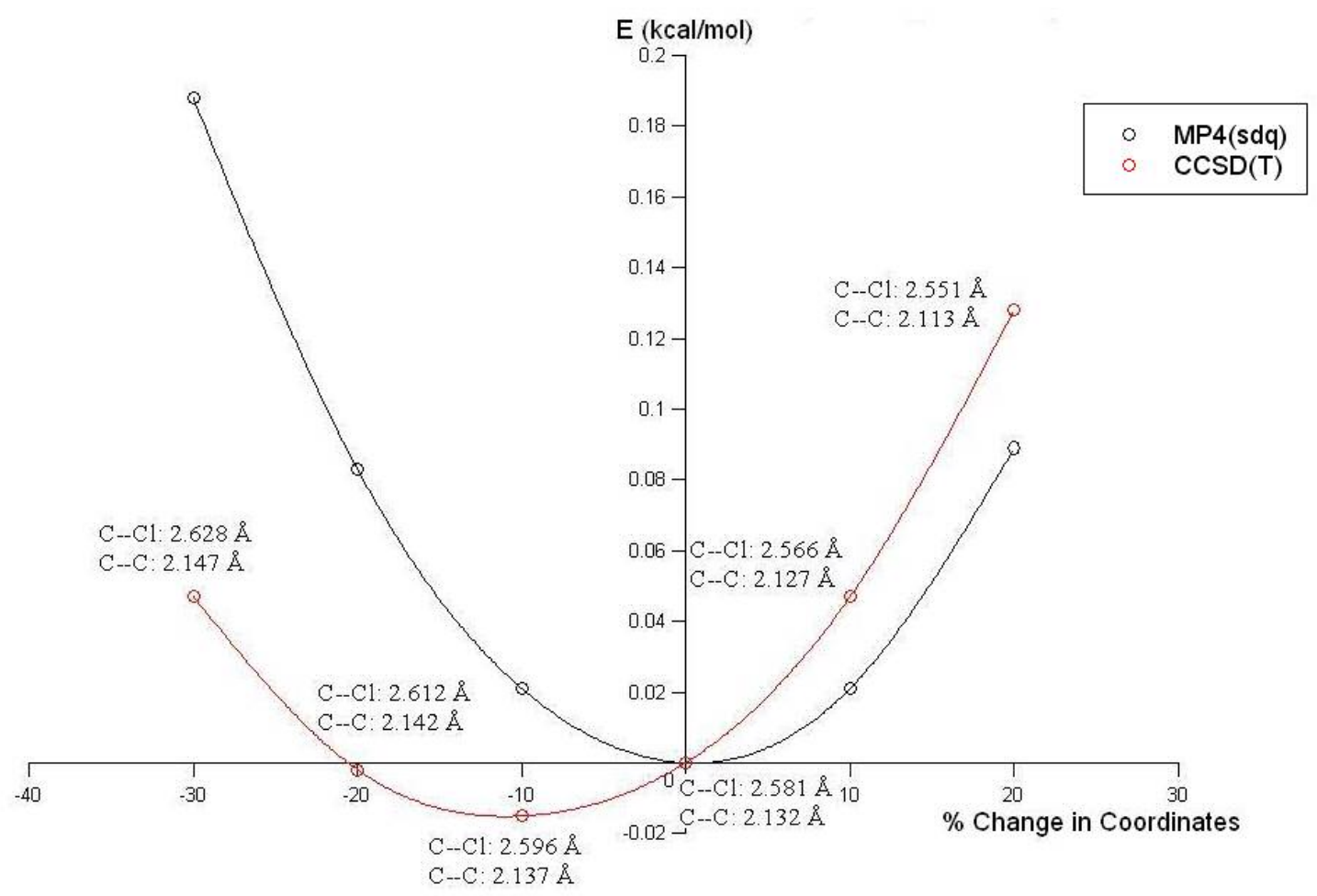

Figure S13. Structure 12 Inversion Process at 6-311+G* Method Comparison on Lowest Positive Frequency $\left(100.70 \mathrm{~cm}^{-1}\right)$ coordinates. 


\section{Calculated Transition-state Energies (Solvated)}

Table S9: Transition-State Energies (Solvated) for 1,2, 6-14

\begin{tabular}{|c|c|c|c|}
\hline \multirow{2}{*}{ Structure } & \multirow{2}{*}{ Direction } & \multicolumn{2}{|c|}{ Transition State Energies (kcal/mol) } \\
\hline & & Own Ground State & Most Stable Ground State \\
\hline \multirow{4}{*}{1} & Inversion A & 21.82 & 24.83 \\
\hline & Inversion B & 21.88 & 23.76 \\
\hline & Retention $\mathrm{C}$ & 24.73 & 24.73 \\
\hline & Retention D & 27.26 & 27.26 \\
\hline \multirow{4}{*}{2} & Inversion $\mathrm{E}$ & 21.37 & 21.37 \\
\hline & Inversion $\mathrm{F}$ & 19.85 & 19.85 \\
\hline & Retention $\mathrm{G}$ & 18.80 & 21.44 \\
\hline & Retention $\mathrm{H}$ & 19.79 & 22.43 \\
\hline \multirow{2}{*}{6} & Inversion & 18.61 & 18.61 \\
\hline & Retention & 26.50 & 28.03 \\
\hline \multirow{2}{*}{7} & Inversion & 13.96 & 13.96 \\
\hline & Retention & 18.04 & 19.91 \\
\hline \multirow{2}{*}{8} & Inversion & 16.13 & 16.13 \\
\hline & Retention & 22.73 & 22.73 \\
\hline \multirow{4}{*}{9} & Inversion $\mathrm{A}$ & 17.46 & 17.46 \\
\hline & Inversion B & 22.93 & 22.93 \\
\hline & Retention $\mathrm{C}$ & 21.33 & 23.09 \\
\hline & Retention D & 25.70 & 27.46 \\
\hline \multirow{2}{*}{10} & Inversion & 19.42 & 19.42 \\
\hline & Retention & 21.31 & 23.78 \\
\hline \multirow{2}{*}{11} & Inversion & 21.73 & 21.73 \\
\hline & Retention & 21.81 & 24.29 \\
\hline \multirow{2}{*}{12} & Inversion & 17.96 & 19.94 \\
\hline & Retention & 20.83 & 20.83 \\
\hline \multirow{2}{*}{13} & Inversion & 17.59 & 17.59 \\
\hline & Retention & 19.59 & 20.94 \\
\hline \multirow{2}{*}{14} & Inversion & 17.24 & 20.61 \\
\hline & Retention & 20.40 & 20.40 \\
\hline
\end{tabular}

\section{Coordinates, ZPVE and Lowest Frequency for all Structures Obtained in this Study}

No significance should be attached to the enantiomer used - in some cases this was a random choice. 
$\underline{\text { Structure } 1}$

(1R)-Retention Ground State MP2/6-311+G*

$\begin{array}{cccc} & \mathrm{X} & \mathrm{Y} & \mathrm{Z} \\ 6 & -0.325984 & -0.227555 & -0.046215 \\ 6 & 1.954226 & -1.375642 & -0.348745 \\ 6 & 1.856726 & 1.088814 & 0.147651 \\ 6 & 2.608145 & -0.015503 & -0.597764 \\ 6 & 0.377850 & 1.126810 & -0.241414 \\ 6 & 0.471427 & -1.349808 & -0.719014 \\ 1 & 2.068572 & -1.645491 & 0.708578 \\ 1 & 1.952199 & 0.935230 & 1.231569 \\ 1 & 2.590027 & 0.203524 & -1.675279 \\ 1 & 0.307057 & 1.280200 & -1.326814 \\ 1 & 0.347868 & -1.183314 & -1.796396 \\ 1 & 2.463844 & -2.155836 & -0.931400 \\ 1 & 2.306445 & 2.067960 & -0.072328 \\ 1 & 3.664811 & -0.035138 & -0.295250 \\ 1 & -0.012137 & -2.304624 & -0.480279 \\ 6 & -1.745484 & -0.168511 & -0.691964 \\ 6 & -2.870427 & -0.501995 & 0.032959 \\ 1 & -3.833911 & -0.466984 & -0.469973 \\ 1 & -2.837169 & -0.789018 & 1.074447 \\ 17 & -0.341710 & -0.608986 & 1.751607 \\ 6 & -0.360342 & 2.279472 & 0.432195 \\ 1 & -0.317165 & 2.193870 & 1.522745 \\ 1 & -1.410492 & 2.287493 & 0.129842 \\ 1 & 0.096013 & 3.235026 & 0.145163 \\ 8 & -1.671715 & 0.199171 & -1.918509\end{array}$

Lowest Frequency $=72.1910 \mathrm{~cm}^{-1}$

Energy $=-885.031365$ Hartrees

Zero Point Correction $=0.214492$ Hartrees 
(1S)-Retention Transition State $\mathbf{C}$ MP2/6-311+G*

$\begin{array}{cccc} & \mathrm{X} & \mathrm{Y} & \mathrm{Z} \\ 6 & -0.447633 & -0.235023 & -0.450133 \\ 6 & 1.893632 & 0.364474 & -1.329657 \\ 6 & 1.658744 & -1.092289 & 0.689960 \\ 6 & 2.399022 & -0.896155 & -0.631148 \\ 6 & 0.143247 & -1.211871 & 0.515221 \\ 6 & 0.393392 & 0.261629 & -1.583515 \\ 1 & 2.083945 & 1.238052 & -0.702143 \\ 1 & 1.856320 & -0.238614 & 1.346922 \\ 1 & 3.479675 & -0.824817 & -0.450170 \\ 1 & -0.083527 & -2.179405 & 0.017836 \\ 1 & 0.223208 & -0.489246 & -2.380523 \\ 1 & 2.408501 & 0.508269 & -2.289707 \\ 1 & 2.015724 & -1.998044 & 1.200497 \\ 1 & 2.244638 & -1.772076 & -1.281098 \\ 1 & -0.023017 & 1.192299 & -1.985263 \\ 6 & -1.889496 & -0.262585 & -0.622371 \\ 6 & -2.345593 & 1.065615 & -0.824847 \\ 1 & -3.360611 & 1.311216 & -0.516894 \\ 1 & -1.670299 & 1.878229 & -1.052368 \\ 17 & 0.373095 & 2.044402 & 1.096841 \\ 6 & -0.564118 & -1.231822 & 1.874679 \\ 1 & -0.119023 & -2.012975 & 2.502394 \\ 1 & -0.421971 & -0.259472 & 2.351750 \\ 1 & -1.628870 & -1.433364 & 1.755027 \\ 8 & -2.599310 & -1.280841 & -0.373961\end{array}$

Lowest Frequency $=-187.6468 \mathrm{~cm}^{-1}$

Energy $=-884.981517$ Hartrees

Zero Point Correction $=0.212437$ Hartrees 
(1S)-Retention Transition State D MP2/6-311+G*

$\begin{array}{cccc} & \mathrm{X} & \mathrm{Y} & \mathrm{Z} \\ 6 & 0.455613 & 0.469822 & 0.171091 \\ 6 & -1.759869 & 1.266660 & -0.852751 \\ 6 & -1.792232 & -0.428402 & 0.991393 \\ 6 & -2.468919 & 0.815216 & 0.420881 \\ 6 & -0.301183 & -0.211910 & 1.278426 \\ 6 & -0.282338 & 1.528058 & -0.587966 \\ 1 & -1.844276 & 0.488020 & -1.615266 \\ 1 & -1.884184 & -1.259979 & 0.286635 \\ 1 & -3.526937 & 0.602860 & 0.217375 \\ 1 & -0.249388 & 0.586349 & 2.053936 \\ 1 & 0.276871 & 1.738229 & -1.505357 \\ 1 & -2.221401 & 2.182912 & -1.246019 \\ 1 & -2.276974 & -0.723080 & 1.933413 \\ 1 & -2.446990 & 1.625213 & 1.167173 \\ 1 & -0.158495 & 2.440644 & 0.024504 \\ 6 & 1.895071 & 0.673465 & 0.171000 \\ 6 & 2.558860 & -0.571812 & 0.047338 \\ 1 & 3.494393 & -0.589137 & -0.509632 \\ 1 & 2.081060 & -1.511915 & 0.272333 \\ 17 & -0.200799 & -1.599503 & -1.669332 \\ 6 & 0.297397 & -1.465994 & 1.910311 \\ 1 & 0.302509 & -2.277553 & 1.177941 \\ 1 & -0.330858 & -1.765639 & 2.756104 \\ 1 & 1.312770 & -1.304259 & 2.280213 \\ 8 & 2.408886 & 1.813532 & -0.026631\end{array}$

Lowest Frequency $=-205.1667 \mathrm{~cm}^{-1}$

Energy $=-884.976053$ Hartrees

Zero Point Correction $=0.212805$ Hartrees 
(1S)-Inversion Ground State $\mathbf{A}$ MP2/6-311+G*

$\begin{array}{cccc} & \mathrm{X} & \mathrm{Y} & \mathrm{Z} \\ 6 & 0.302667 & -0.270064 & 0.063195 \\ 6 & -2.054964 & -1.272663 & 0.273342 \\ 6 & -1.779459 & 1.110173 & -0.487444 \\ 6 & -2.618898 & -0.161625 & -0.612754 \\ 6 & -0.297774 & 0.862786 & -0.797399 \\ 6 & -0.578438 & -1.519178 & -0.041666 \\ 1 & -2.171128 & -0.998266 & 1.327789 \\ 1 & -1.870645 & 1.518332 & 0.527753 \\ 1 & -3.667618 & 0.043483 & -0.355623 \\ 1 & -0.228900 & 0.464950 & -1.823240 \\ 1 & -0.504431 & -1.882861 & -1.072587 \\ 1 & -2.619757 & -2.203267 & 0.121669 \\ 1 & -2.156844 & 1.882172 & -1.173359 \\ 1 & -2.611018 & -0.495307 & -1.661202 \\ 1 & -0.158500 & -2.298277 & 0.603215 \\ 6 & 1.810184 & -0.492973 & -0.315505 \\ 6 & 2.067355 & -1.447199 & -1.295480 \\ 1 & 3.093382 & -1.559415 & -1.637936 \\ 1 & 1.325657 & -2.117913 & -1.709524 \\ 17 & 0.247523 & 0.238018 & 1.821782 \\ 6 & 0.496491 & 2.165718 & -0.748987 \\ 1 & 1.536413 & 2.003760 & -1.034527 \\ 1 & 0.038605 & 2.899960 & -1.424623 \\ 1 & 0.497038 & 2.578621 & 0.264158 \\ 8 & 2.650857 & 0.283483 & 0.231741\end{array}$

Lowest Frequency $=34.1986 \mathrm{~cm}^{-1}$

Energy $=-885.022981$ Hartrees

Zero Point Correction $=0.214344$ Hartrees 
(1R)-Inversion Ground State B MP2/6-311+G*

$\begin{array}{cccc} & \mathrm{X} & \mathrm{Y} & \mathrm{Z} \\ 6 & -0.343483 & -0.153883 & -0.038586 \\ 6 & 1.819207 & -1.509731 & -0.410908 \\ 6 & 1.959119 & 0.958616 & -0.030386 \\ 6 & 2.563329 & -0.227146 & -0.780124 \\ 6 & 0.457608 & 1.132240 & -0.298679 \\ 6 & 0.327104 & -1.365409 & -0.696234 \\ 1 & 1.972817 & -1.728462 & 0.653298 \\ 1 & 2.113870 & 0.823330 & 1.048581 \\ 1 & 3.635108 & -0.316908 & -0.553740 \\ 1 & 0.339570 & 1.312491 & -1.376998 \\ 1 & -0.239465 & -2.250625 & -0.393985 \\ 1 & 2.220292 & -2.364547 & -0.972918 \\ 1 & 2.471955 & 1.891393 & -0.307233 \\ 1 & 2.480925 & -0.056135 & -1.863785 \\ 1 & 0.167638 & -1.241035 & -1.778076 \\ 6 & -1.840029 & -0.187169 & -0.507952 \\ 6 & -2.265969 & 0.800913 & -1.387632 \\ 1 & -3.268028 & 0.718158 & -1.802135 \\ 1 & -1.677371 & 1.666946 & -1.661681 \\ 17 & -0.321737 & -0.448263 & 1.775201 \\ 6 & -0.058529 & 2.357025 & 0.462179 \\ 1 & 0.339913 & 3.274012 & 0.009888 \\ 1 & -1.148133 & 2.403435 & 0.446227 \\ 1 & 0.270882 & 2.324336 & 1.505049 \\ 8 & -2.490073 & -1.208580 & -0.125123\end{array}$

Lowest Frequency $=70.1388 \mathrm{~cm}^{-1}$

Energy $=-885.023439$ Hartrees

Zero Point Correction $=0.214386$ Hartrees 
(1S)-Inversion Transition State $\mathbf{A}$ MP2/6-311+G*

$\begin{array}{cccc} & \mathrm{X} & \mathrm{Y} & \mathrm{Z} \\ 6 & -0.386757 & -0.488445 & -0.123842 \\ 6 & 2.017079 & -0.684093 & -1.001059 \\ 6 & 1.592367 & 0.262735 & 1.291953 \\ 6 & 2.513358 & -0.608309 & 0.440909 \\ 6 & 0.139625 & -0.238517 & 1.282687 \\ 6 & 0.579606 & -1.214549 & -1.054776 \\ 1 & 2.057296 & 0.303170 & -1.461703 \\ 1 & 1.607751 & 1.292994 & 0.926666 \\ 1 & 3.536275 & -0.208769 & 0.466925 \\ 1 & 0.163627 & -1.242311 & 1.747103 \\ 1 & 0.633434 & -2.272672 & -0.760015 \\ 1 & 2.654125 & -1.364213 & -1.585155 \\ 1 & 1.936792 & 0.265697 & 2.336760 \\ 1 & 2.560142 & -1.621891 & 0.871180 \\ 1 & 0.198382 & -1.166035 & -2.079610 \\ 6 & -1.827441 & -0.419501 & -0.364876 \\ 6 & -2.009111 & -1.840534 & -0.405112 \\ 1 & -2.815261 & -2.264409 & 0.195143 \\ 1 & -1.434946 & -2.505259 & -1.033069 \\ 17 & 0.104170 & 1.875962 & -1.136602 \\ 6 & -0.750510 & 0.661952 & 2.135698 \\ 1 & -0.289014 & 0.797612 & 3.122170 \\ 1 & -0.866932 & 1.632349 & 1.648839 \\ 1 & -1.747049 & 0.236814 & 2.272345 \\ 8 & -2.646852 & 0.455142 & -0.069355\end{array}$

Lowest Frequency $=-336.6806 \mathrm{~cm}^{-1}$

Energy $=-884.979256$ Hartrees

Zero Point Correction $=0.213046$ Hartrees 
(1R)-Inversion Transition State $\mathbf{B}$ MP2/6-311+G*

$\begin{array}{cccc} & \mathrm{X} & \mathrm{Y} & \mathrm{Z} \\ 6 & -0.464958 & 0.185266 & -0.360749 \\ 6 & 1.698163 & -0.800464 & -1.362027 \\ 6 & 1.837440 & 0.903747 & 0.462965 \\ 6 & 2.412344 & 0.458953 & -0.878971 \\ 6 & 0.345917 & 1.262272 & 0.363426 \\ 6 & 0.193178 & -0.569690 & -1.502608 \\ 1 & 1.861801 & -1.607400 & -0.644107 \\ 1 & 1.968034 & 0.110408 & 1.203563 \\ 1 & 3.490421 & 0.271010 & -0.782778 \\ 1 & 0.324089 & 2.168398 & -0.262741 \\ 1 & -0.337376 & -1.519061 & -1.615366 \\ 1 & 2.099094 & -1.122090 & -2.333786 \\ 1 & 2.363395 & 1.799180 & 0.826004 \\ 1 & 2.297253 & 1.266116 & -1.620351 \\ 1 & 0.012430 & 0.021682 & -2.415658 \\ 6 & -1.919579 & 0.050283 & -0.336791 \\ 6 & -2.184282 & 1.385203 & -0.781819 \\ 1 & -2.857491 & 1.505285 & -1.631261 \\ 1 & -1.767298 & 2.272577 & -0.331270 \\ 17 & 0.122408 & -1.730473 & 1.371726 \\ 6 & -0.202350 & 1.606650 & 1.751263 \\ 1 & 0.390684 & 2.418548 & 2.191231 \\ 1 & -1.247846 & 1.921767 & 1.725299 \\ 1 & -0.131173 & 0.720170 & 2.384633 \\ 8 & -2.605275 & -0.962733 & -0.517613\end{array}$

Lowest Frequency $=-309.6671 \mathrm{~cm}^{-1}$

Energy $=-884.979734$ Hartrees

Zero Point Correction $=0.212968$ Hartrees 
$\underline{\text { Structure } 2}$

(1R)-Retention Ground State MP2/6-311+G*

$\begin{array}{cccc} & \mathrm{X} & \mathrm{Y} & \mathrm{Z} \\ 6 & 2.566423 & -0.375324 & 0.171268 \\ 6 & 1.802411 & -0.273620 & -1.151033 \\ 6 & 0.313097 & -0.625901 & -1.001640 \\ 6 & -0.367671 & 0.197729 & 0.113809 \\ 6 & 0.441888 & 0.183821 & 1.410448 \\ 6 & 1.921038 & 0.523391 & 1.227691 \\ 6 & 0.117058 & -2.131789 & -0.787031 \\ 6 & -1.820940 & -0.326273 & 0.364927 \\ 8 & -1.882123 & -1.206274 & 1.291780 \\ 6 & -2.829933 & 0.088246 & -0.484669 \\ 17 & -0.375225 & 1.947029 & -0.469179 \\ 1 & -3.823475 & -0.334143 & -0.355334 \\ 1 & -2.693015 & 0.879915 & -1.210257 \\ 1 & -0.037267 & 0.868877 & 2.118899 \\ 1 & 0.320927 & -0.817911 & 1.833803 \\ 1 & 2.032653 & 1.572850 & 0.927364 \\ 1 & 1.895669 & 0.749792 & -1.533811 \\ 1 & 3.617741 & -0.093480 & 0.018560 \\ 1 & 2.251031 & -0.936270 & -1.905231 \\ 1 & 2.571400 & -1.414333 & 0.526384 \\ 1 & -0.205400 & -0.361484 & -1.932846 \\ 1 & 2.441357 & 0.413354 & 2.189327 \\ 1 & 0.625761 & -2.678711 & -1.591979 \\ 1 & 0.521682 & -2.472559 & 0.169298 \\ 1 & -0.943488 & -2.386881 & -0.795007\end{array}$

Lowest Frequency $=61.8281 \mathrm{~cm}^{-1}$

Energy $=-885.025155$ Hartrees

Zero Point Correction $=0.214676$ Hartrees 
(1R)-Retention Transition State $\mathbf{G}$ MP2/6-311+G*

$\begin{array}{cccc} & \mathrm{X} & \mathrm{Y} & \mathrm{Z} \\ 6 & 2.443930 & -0.575337 & 0.347350 \\ 6 & 1.775984 & -0.309507 & -1.002977 \\ 6 & 0.304407 & -0.740168 & -1.026534 \\ 6 & -0.474996 & -0.309132 & 0.180271 \\ 6 & 0.221063 & -0.262774 & 1.495461 \\ 6 & 1.688284 & 0.152806 & 1.458397 \\ 6 & 0.171548 & -2.282091 & -1.109036 \\ 6 & -1.914166 & -0.441328 & 0.249071 \\ 8 & -2.523490 & -0.832048 & 1.284273 \\ 6 & -2.487731 & 0.230856 & -0.866080 \\ 17 & 0.073324 & 2.514712 & -0.379351 \\ 1 & -3.484394 & 0.653654 & -0.754583 \\ 1 & -1.909178 & 0.519745 & -1.731561 \\ 1 & -0.377670 & 0.354214 & 2.173775 \\ 1 & 0.116220 & -1.289669 & 1.897999 \\ 1 & 1.730757 & 1.230833 & 1.279353 \\ 1 & 1.812279 & 0.762292 & -1.212759 \\ 1 & 3.486034 & -0.231406 & 0.313012 \\ 1 & 2.309808 & -0.836645 & -1.806887 \\ 1 & 2.481258 & -1.654247 & 0.559394 \\ 1 & -0.165349 & -0.336257 & -1.930559 \\ 1 & 2.147561 & -0.050763 & 2.435331 \\ 1 & 0.747527 & -2.653051 & -1.965398 \\ 1 & 0.551878 & -2.770226 & -0.206538 \\ 1 & -0.875270 & -2.572136 & -1.231335\end{array}$

Lowest Frequency $=-163.1881 \mathrm{~cm}^{-1}$

Energy $=-884.979931$ Hartrees

Zero Point Correction $=0.212617$ Hartrees 
(1S)-Retention Transition State $\mathbf{H}$ MP2/6-311+G*

$\begin{array}{cccc} & \mathrm{X} & \mathrm{Y} & \mathrm{Z} \\ 6 & -2.514950 & -0.164289 & 0.313547 \\ 6 & -1.752801 & -0.370993 & -0.997421 \\ 6 & -0.365797 & -0.985149 & -0.784592 \\ 6 & 0.428081 & -0.334939 & 0.301406 \\ 6 & -0.318929 & 0.065917 & 1.533459 \\ 6 & -1.695950 & 0.686286 & 1.284364 \\ 6 & -0.456092 & -2.491034 & -0.423307 \\ 6 & 1.860176 & -0.555342 & 0.266823 \\ 8 & 2.408403 & -1.434581 & -0.457935 \\ 6 & 2.532244 & 0.525497 & 0.904838 \\ 17 & 0.246762 & 2.332601 & -0.894395 \\ 1 & 3.553534 & 0.740011 & 0.597287 \\ 1 & 2.021852 & 1.260986 & 1.509176 \\ 1 & 0.291209 & 0.694566 & 2.188232 \\ 1 & -0.457788 & -0.879821 & 2.092645 \\ 1 & -1.546888 & 1.684133 & 0.865004 \\ 1 & -1.618339 & 0.596427 & -1.487884 \\ 1 & -3.474196 & 0.328008 & 0.106628 \\ 1 & -2.326944 & -1.022300 & -1.672402 \\ 1 & -2.758472 & -1.131678 & 0.777069 \\ 1 & 0.231375 & -0.914440 & -1.701430 \\ 1 & -2.222233 & 0.786455 & 2.244015 \\ 1 & -0.979347 & -3.017316 & -1.230586 \\ 1 & 0.546298 & -2.908538 & -0.317036 \\ 1 & -1.018133 & -2.649780 & 0.502762\end{array}$

Lowest Frequency $=-162.9751 \mathrm{~cm}^{-1}$

Energy $=-884.979123$ Hartrees

Zero Point Correction $=0.212994$ Hartrees 
(1S)-Inversion Ground State MP2/6-311+G*

$\begin{array}{cccc} & \mathrm{X} & \mathrm{Y} & \mathrm{Z} \\ 6 & 2.577931 & 0.312143 & 0.336136 \\ 6 & 1.840602 & 0.567696 & -0.980766 \\ 6 & 0.354125 & 0.884553 & -0.769868 \\ 6 & -0.362354 & -0.191575 & 0.064725 \\ 6 & 0.409183 & -0.513990 & 1.345263 \\ 6 & 1.894214 & -0.811207 & 1.117025 \\ 6 & 0.157210 & 2.269457 & -0.138877 \\ 6 & -1.862187 & 0.204345 & 0.220646 \\ 8 & -2.419805 & 0.588513 & -0.858098 \\ 6 & -2.412460 & 0.155812 & 1.491846 \\ 17 & -0.360370 & -1.741193 & -0.939244 \\ 1 & -3.455704 & 0.437301 & 1.615782 \\ 1 & -1.881180 & -0.217922 & 2.358293 \\ 1 & 0.307921 & 0.350858 & 2.012396 \\ 1 & -0.074808 & -1.358641 & 1.845019 \\ 1 & 1.998850 & -1.752610 & 0.564688 \\ 1 & 1.934100 & -0.320213 & -1.617597 \\ 1 & 3.627386 & 0.053025 & 0.137954 \\ 1 & 2.310320 & 1.399146 & -1.525688 \\ 1 & 2.594247 & 1.226772 & 0.943927 \\ 1 & -0.162825 & 0.899449 & -1.735007 \\ 1 & 2.388331 & -0.956893 & 2.087813 \\ 1 & -0.905752 & 2.514341 & -0.110459 \\ 1 & 0.548889 & 2.330201 & 0.881148 \\ 1 & 0.677377 & 3.023968 & -0.743109\end{array}$

Lowest Frequency $=86.1205 \mathrm{~cm}^{-1}$

Energy $=-885.025808$ Hartrees

Zero Point Correction $=0.214840$ Hartrees 
(1S)-Inversion Transition State $\mathbf{E}$ MP2/6-311+G*

$\begin{array}{cccc} & \mathrm{X} & \mathrm{Y} & \mathrm{Z} \\ 6 & -2.461831 & 0.201767 & 0.470208 \\ 6 & -1.816589 & -0.004292 & -0.900587 \\ 6 & -0.546681 & -0.884507 & -0.847400 \\ 6 & 0.412347 & -0.394514 & 0.218957 \\ 6 & -0.200210 & -0.092649 & 1.560102 \\ 6 & -1.453429 & 0.788775 & 1.455387 \\ 6 & -0.980536 & -2.346404 & -0.597458 \\ 6 & 1.868407 & -0.401622 & 0.203046 \\ 8 & 2.615629 & 0.204332 & 0.985941 \\ 6 & 2.132234 & -1.653232 & -0.439622 \\ 17 & 0.550819 & 2.215312 & -0.936935 \\ 1 & 2.917146 & -2.292054 & -0.036040 \\ 1 & 1.621911 & -1.964416 & -1.340000 \\ 1 & 0.564883 & 0.383470 & 2.178132 \\ 1 & -0.477008 & -1.046981 & 2.039872 \\ 1 & -1.139170 & 1.777820 & 1.112673 \\ 1 & -2.530413 & -0.478176 & -1.590035 \\ 1 & -2.865631 & -0.742929 & 0.863313 \\ 1 & -1.532005 & 0.964597 & -1.312049 \\ 1 & -3.317452 & 0.881876 & 0.363694 \\ 1 & -0.059173 & -0.809990 & -1.826487 \\ 1 & -1.901907 & 0.892693 & 2.453474 \\ 1 & -1.408920 & -2.468324 & 0.401307 \\ 1 & -0.136306 & -3.034577 & -0.677377 \\ 1 & -1.747181 & -2.637913 & -1.325907\end{array}$

Lowest Frequency $=-187.6829 \mathrm{~cm}^{-1}$

Energy $=-884.974594$ Hartrees

Zero Point Correction $=0.213259$ Hartrees 
(1S)-Inversion Transition State $\mathbf{F}$ MP2/6-311+G*

$\begin{array}{cccc} & \mathrm{X} & \mathrm{Y} & \mathrm{Z} \\ 6 & -2.352342 & -0.898385 & 0.168905 \\ 6 & -1.567497 & -0.685962 & -1.124405 \\ 6 & -0.048824 & -0.817827 & -0.939155 \\ 6 & 0.469886 & -0.111164 & 0.297310 \\ 6 & -0.423583 & -0.030563 & 1.527680 \\ 6 & -1.914395 & 0.129117 & 1.209217 \\ 6 & 0.393217 & -2.292579 & -0.909896 \\ 6 & 1.894151 & 0.176682 & 0.241123 \\ 8 & 2.583856 & 0.462638 & -0.744573 \\ 6 & 2.232838 & -0.677225 & 1.340531 \\ 17 & -0.309666 & 2.394002 & -0.488264 \\ 1 & 3.047911 & -1.388966 & 1.205182 \\ 1 & 1.762379 & -0.611305 & 2.310835 \\ 1 & -0.335065 & -0.965597 & 2.099077 \\ 1 & -0.083196 & 0.791077 & 2.165061 \\ 1 & -2.097380 & 1.137623 & 0.835451 \\ 1 & -1.770145 & 0.323139 & -1.493488 \\ 1 & -3.427701 & -0.799618 & -0.031396 \\ 1 & -1.888678 & -1.401622 & -1.895316 \\ 1 & -2.199496 & -1.918031 & 0.555309 \\ 1 & 0.448967 & -0.330249 & -1.784772 \\ 1 & -2.478758 & -0.002529 & 2.143444 \\ 1 & 1.483889 & -2.364057 & -0.874193 \\ 1 & -0.010212 & -2.813313 & -0.034328 \\ 1 & 0.040258 & -2.808252 & -1.811656\end{array}$

Lowest Frequency $=-243.6716 \mathrm{~cm}^{-1}$

Energy $=-884.980532$ Hartrees

Zero Point Correction $=0.213310$ Hartrees

\section{$\underline{\text { Structure } 6}$}

Retention Ground State MP2/6-311+G*

$\begin{array}{cccc} & \mathrm{X} & \mathrm{Y} & \mathrm{Z} \\ 6 & 1.108650 & 0.033089 & -0.000046 \\ 8 & 2.109197 & -0.765263 & -0.000026 \\ 6 & 1.073076 & 1.410042 & -0.000076 \\ 1 & 0.141473 & 1.961213 & -0.000111 \\ 1 & 2.009020 & 1.961769 & -0.000148 \\ 6 & -0.191403 & -0.793092 & 0.000053 \\ 17 & -1.798442 & 0.067892 & 0.000040 \\ 1 & -0.196272 & -1.427703 & -0.886579 \\ 1 & -0.196223 & -1.427582 & 0.886774\end{array}$

Lowest Frequency $=61.4796 \mathrm{~cm}^{-1}$

Energy $=-651.088906$ Hartrees

Zero Point Correction $=0.062320$ Hartrees 
Retention Transition State MP2/6-311+G*

$\begin{array}{cccc} & \mathrm{X} & \mathrm{Y} & \mathrm{Z} \\ 6 & 1.437927 & -0.064242 & 0.120005 \\ 8 & 2.298939 & -0.710229 & -0.517309 \\ 6 & 1.155686 & 1.337806 & 0.066817 \\ 1 & 1.417913 & 1.913910 & -0.818723 \\ 1 & 0.569141 & 1.819318 & 0.840772 \\ 6 & 0.251986 & -0.576380 & 0.749616 \\ 17 & -2.185979 & -0.033809 & -0.212551 \\ 1 & -0.169524 & -0.101490 & 1.625483 \\ 1 & -0.120994 & -1.558256 & 0.485686\end{array}$

Lowest Frequency $=-328.6640 \mathrm{~cm}^{-1}$

Energy $=-651.036586$ Hartrees

Zero Point Correction $=0.060314$ Hartrees

Inversion Ground State

MP2/6-311+G*

$\begin{array}{cccc} & \mathrm{X} & \mathrm{Y} & \mathrm{Z} \\ 6 & -1.033005 & 0.123173 & 0.069413 \\ 8 & -1.155393 & 1.386729 & 0.016000 \\ 6 & -1.933591 & -0.864487 & -0.314421 \\ 1 & -1.672285 & -1.917831 & -0.278860 \\ 1 & -2.891666 & -0.586983 & -0.746770 \\ 6 & 0.249239 & -0.414969 & 0.713029 \\ 17 & 1.732783 & -0.013210 & -0.259586 \\ 1 & 0.248483 & -1.499796 & 0.825632 \\ 1 & 0.405450 & 0.073041 & 1.676841\end{array}$

Lowest Frequency $=84.1994 \mathrm{~cm}^{-1}$

Energy $=-651.088796$ Hartrees

Zero Point Correction $=0.062889$ Hartrees

Inversion Transition State

MP2/6-311+G*

\begin{tabular}{cc} 
& $\mathrm{X}$ \\
6 & -1.20879 \\
8 & -1.39553 \\
6 & -2.12534 \\
1 & -1.929804 \\
1 & -3.10771 \\
6 & -0.16987 \\
17 & 2.178482 \\
1 & 0.091305 \\
1 & 0.100418 \\
\multicolumn{2}{c}{} \\
Frequency = -390.1393 $\mathrm{cm}^{-1}$ \\
$=-651.051833$ Hartrees \\
Point Correction = 0.060865 Hartrees
\end{tabular}

$\begin{array}{cc}\mathrm{Y} & \mathrm{Z} \\ 0.242927 & -0.115695 \\ 1.454837 & 0.018302 \\ -0.856423 & -0.182251 \\ -1.745699 & -0.767027 \\ -0.748649 & 0.275753 \\ -0.634515 & 0.390909 \\ 0.025279 & -0.089547 \\ -1.542087 & -0.125738 \\ -0.543945 & 1.435127\end{array}$

Lowest Frequency $=-390.1393 \mathrm{~cm}^{-1}$

Energy $=-651.051833$ Hartrees

Zero Point Correction $=0.060865$ Hartrees 
$\underline{\text { Structure } 7}$

Retention Ground State MP2/6-311+G*

$\begin{array}{cccc} & \mathrm{X} & \mathrm{Y} & \mathrm{Z} \\ 6 & -2.529389 & -1.004639 & -0.004483 \\ 6 & -1.049325 & -0.739864 & 0.010186 \\ 6 & -0.570290 & 0.550992 & 0.005227 \\ 8 & -1.223325 & 1.658792 & -0.005523 \\ 6 & 0.935108 & 0.847419 & 0.005950 \\ 17 & 2.110057 & -0.546805 & -0.003867 \\ 1 & -0.359219 & -1.577207 & 0.030291 \\ 1 & 1.171183 & 1.440998 & -0.878120 \\ 1 & 1.176529 & 1.430747 & 0.895453 \\ 1 & -2.794521 & -1.834902 & -0.673979 \\ 1 & -2.944422 & -1.251260 & 0.986291 \\ 1 & -3.050541 & -0.106471 & -0.351303\end{array}$

Lowest Frequency $=49.2140 \mathrm{~cm}^{-1}$

Energy $=-690.271736$ Hartrees

Zero Point Correction $=0.091079$ Hartrees

Retention Transition State MP2/6-311+G*

$\begin{array}{cccc} & \mathrm{X} & \mathrm{Y} & \mathrm{Z} \\ 6 & 1.785267 & 1.607718 & -0.164374 \\ 6 & 1.093758 & 0.570048 & 0.645830 \\ 6 & 1.005198 & -0.781236 & 0.175855 \\ 8 & 1.730524 & -1.321638 & -0.693832 \\ 6 & -0.306175 & -1.182914 & 0.590305 \\ 17 & -2.366963 & 0.333628 & -0.230215 \\ 1 & 0.545942 & 0.856043 & 1.540040 \\ 1 & -0.690709 & -0.919981 & 1.566813 \\ 1 & -0.864106 & -1.907421 & 0.009065 \\ 1 & 1.303222 & 2.583840 & -0.064365 \\ 1 & 2.836973 & 1.710600 & 0.140937 \\ 1 & 1.794581 & 1.296651 & -1.213875\end{array}$

Lowest Frequency $=-193.6484 \mathrm{~cm}^{-1}$

Energy $=-690.228649$ Hartrees

Zero Point Correction $=0.089070$ Hartrees 
Inversion Ground State MP2/6-311+G*

$\begin{array}{cccc} & \mathrm{X} & \mathrm{Y} & \mathrm{Z} \\ 6 & -2.857767 & -0.283604 & -0.359874 \\ 6 & -1.486095 & -0.696020 & 0.094371 \\ 6 & -0.480086 & 0.248649 & 0.257726 \\ 8 & -0.552187 & 1.509366 & 0.068498 \\ 6 & 0.831940 & -0.301756 & 0.800035 \\ 17 & 2.198451 & -0.151455 & -0.400497 \\ 1 & -1.265590 & -1.753853 & 0.227704 \\ 1 & 0.775518 & -1.361007 & 1.055566 \\ 1 & 1.156007 & 0.288707 & 1.659323 \\ 1 & -2.964653 & -0.251298 & -1.456319 \\ 1 & -3.637247 & -0.956191 & 0.021745 \\ 1 & -3.068159 & 0.729835 & -0.001100\end{array}$

Lowest Frequency $=66.0404 \mathrm{~cm}^{-1}$

Energy $=-690.272293$ Hartrees

Zero Point Correction $=0.091371$ Hartrees

Inversion Transition State MP2/6-311+G*

\begin{tabular}{cc} 
& $\mathrm{X}$ \\
6 & -3.043335 \\
6 & -1.63783 \\
6 & -0.641008 \\
8 & -0.777262 \\
6 & 0.307355 \\
17 & 2.756982 \\
1 & -1.424536 \\
1 & 0.522019 \\
1 & 0.571632 \\
1 & -3.660469 \\
1 & -3.527813 \\
1 & -3.042526 \\
\multicolumn{2}{c}{} \\
Frequency $=-289.2720 \mathrm{~cm}^{-1}$ \\
$=-690.238127$ Hartrees \\
Point Correction = 0.089559 Hartrees
\end{tabular}

$Y$
-0.372038
-0.622014
0.395251
1.613500
-0.546263
-0.101128
-1.517456
-1.477695
-0.448904
0.039069
-1.286513
0.373058

Lowest Frequency $=-289.2720 \mathrm{~cm}^{-1}$

Energy $=-690.238127$ Hartrees

Zero Point Correction $=0.089559$ Hartrees 


\section{$\underline{\text { Structure } 8}$}

Retention/Inversion Ground State MP2/6-311+G*

$\begin{array}{cc} & \mathrm{X} \\ 6 & -0.616649 \\ 8 & -0.478218 \\ 6 & -1.692204 \\ 6 & -1.730730 \\ 1 & -2.533253 \\ 6 & 0.536994 \\ 17 & 1.845997 \\ 1 & 1.023876 \\ 1 & 0.236885 \\ 1 & -2.557383 \\ 1 & -0.819402 \\ 1 & -1.891398\end{array}$

$\mathrm{Y}$
0.815626
2.063195
-0.011585
-1.504608
0.442962
0.157279
-0.478947
0.916979
-0.697147
-1.951358
-2.020055
-1.795119

Lowest Frequency $=54.2287 \mathrm{~cm}^{-1}$

Energy $=-690.264827$ Hartrees

Zero Point Correction $=0.091387$ Hartrees

Retention Transition State MP2/6-311+G*

$\begin{array}{cccc} & \mathrm{X} & \mathrm{Y} & \mathrm{Z} \\ 6 & 1.364622 & -0.446371 & 0.086965 \\ 8 & 2.238578 & -1.244366 & -0.334331 \\ 6 & 1.156716 & 0.892723 & -0.397516 \\ 6 & 0.353720 & 1.987120 & 0.228285 \\ 1 & 1.531307 & 1.064559 & -1.410179 \\ 6 & 0.190750 & -0.793314 & 0.831569 \\ 17 & -2.225802 & -0.342217 & -0.232300 \\ 1 & -0.143893 & -1.824367 & 0.843716 \\ 1 & -0.252928 & -0.120745 & 1.551773 \\ 1 & 0.705052 & 2.963232 & -0.121121 \\ 1 & -0.703156 & 1.871143 & -0.057031 \\ 1 & 0.398788 & 1.977858 & 1.320773\end{array}$

Lowest Frequency $=-242.3754 \mathrm{~cm}^{-1}$

Energy $=-690.222381$ Hartrees

Zero Point Correction $=0.088955$ Hartrees 
Inversion Transition State MP2/6-311+G*

\begin{tabular}{lc} 
& $\mathrm{X}$ \\
6 & -0.72011 \\
8 & -0.59777 \\
6 & -1.899603 \\
6 & -2.19668 \\
1 & -2.72562 \\
6 & 0.14151 \\
17 & 2.45731 \\
1 & 0.55611 \\
1 & 0.08713 \\
1 & -2.49127 \\
1 & -3.02721 \\
1 & -1.34188 \\
\multicolumn{2}{c}{} \\
Frequency $=-337.1679 \mathrm{~cm}^{-1}$ \\
$=-690.231480$ Hartrees \\
Point Correction $=0.089565$ Hartrees $^{-}$
\end{tabular}

Lowest Frequency $=-337.1679 \mathrm{~cm}^{-1}$

Energy $=-690.231480$ Hartrees

Zero Point Correction $=0.089565$ Hartrees

\section{$\underline{\text { Structure } 9}$}

(S)-Retention Ground State MP2/6-311+G*

$\begin{array}{cccc} & \mathrm{X} & \mathrm{Y} & \mathrm{Z} \\ 6 & 1.171589 & -1.504154 & 0.517983 \\ 6 & 1.148739 & -0.267373 & -0.092065 \\ 6 & -0.186246 & 0.472267 & -0.330765 \\ 17 & -1.717254 & -0.511605 & -0.162042 \\ 8 & 2.124418 & 0.464265 & -0.484826 \\ 1 & 0.266925 & -2.031923 & 0.790135 \\ 1 & 2.130999 & -1.980420 & 0.702047 \\ 1 & -0.185339 & 0.792610 & -1.375490 \\ 6 & -0.307909 & 1.680469 & 0.584983 \\ 1 & -1.189974 & 2.283645 & 0.346638 \\ 1 & -0.376410 & 1.349107 & 1.625100 \\ 1 & 0.594741 & 2.282899 & 0.464086\end{array}$

Lowest Frequency $=69.5369 \mathrm{~cm}^{-1}$

Energy $=-690.276614$ Hartrees

Zero Point Correction $=0.091250$ Hartrees 
(S)-Retention Transition State $\mathbf{C}$ MP2/6-311+G*

$\begin{array}{cccc} & \mathrm{X} & \mathrm{Y} & \mathrm{Z} \\ 6 & -1.171088 & -1.666099 & 0.259977 \\ 6 & -1.455970 & -0.280674 & 0.084705 \\ 6 & -0.308506 & 0.422556 & 0.598991 \\ 17 & 2.269331 & -0.333328 & -0.219002 \\ 8 & -2.369576 & 0.242488 & -0.604295 \\ 1 & -1.601638 & -2.402354 & -0.416374 \\ 1 & -0.426670 & -1.992214 & 0.977049 \\ 1 & 0.245947 & 0.014017 & 1.434376 \\ 6 & -0.036174 & 1.801610 & 0.143354 \\ 1 & 0.927011 & 2.165595 & 0.492162 \\ 1 & -0.073096 & 1.839947 & -0.948253 \\ 1 & -0.863139 & 2.437322 & 0.496274\end{array}$

Lowest Frequency $=-186.2077 \mathrm{~cm}^{-1}$

Energy $=-690.230029$ Hartrees

Zero Point Correction $=0.088946$ Hartrees

(R)-Retention Transition State D MP2/6-311+G*

$\begin{array}{cccc} & \mathrm{X} & \mathrm{Y} & \mathrm{Z} \\ 6 & -1.197052 & -0.546762 & 1.293256 \\ 6 & -1.458668 & -0.170326 & -0.056325 \\ 6 & -0.284466 & 0.561973 & -0.460368 \\ 17 & 2.139988 & -0.604998 & -0.111773 \\ 8 & -2.315671 & -0.652925 & -0.837300 \\ 1 & -1.508960 & -1.532702 & 1.632467 \\ 1 & -0.554516 & 0.033581 & 1.940939 \\ 1 & 0.074595 & 0.352493 & -1.463640 \\ 6 & 0.166556 & 1.840108 & 0.156740 \\ 1 & 1.229429 & 2.001685 & -0.012987 \\ 1 & -0.047757 & 1.893537 & 1.226388 \\ 1 & -0.405437 & 2.649818 & -0.324436\end{array}$

Lowest Frequency $=-304.1499 \mathrm{~cm}^{-1}$

Energy $=-690.222964$ Hartrees

Zero Point Correction $=0.088915$ Hartrees 
(S)-Inversion Ground State MP2/6-311+G*

\begin{tabular}{cc} 
& $\mathrm{X}$ \\
6 & 2.04222 \\
6 & 1.11995 \\
6 & -0.22755 \\
17 & -1.56327 \\
8 & 1.25854 \\
1 & 1.764643 \\
1 & 3.03655 \\
1 & -0.214109 \\
6 & -0.60767 \\
1 & -1.57722 \\
1 & -0.63015 \\
1 & 0.16595 \\
\multicolumn{2}{|c}{} \\
Frequency $=77.4440 \mathrm{~cm}^{-1}$ & \\
$=-690.277780$ Hartrees &
\end{tabular}

Lowest Frequency $=77.4440 \mathrm{~cm}^{-1}$

Energy $=-690.277780$ Hartrees

Zero Point Correction $=0.091331$ Hartrees

(S)-Inversion Transition State A MP2/6-311+G*

$\begin{array}{cccc} & \mathrm{X} & \mathrm{Y} & \mathrm{Z} \\ 6 & -2.099104 & -0.206893 & 1.026412 \\ 6 & -1.238925 & -0.461954 & -0.087634 \\ 6 & -0.211387 & 0.435381 & 0.398120 \\ 17 & 2.176642 & -0.472842 & 0.115659 \\ 8 & -1.504330 & -0.808955 & -1.247079 \\ 1 & -1.769081 & -0.317171 & 2.051407 \\ 1 & -3.147202 & 0.029770 & 0.843457 \\ 1 & 0.117090 & 0.364583 & 1.422240 \\ 6 & 0.100638 & 1.660226 & -0.410579 \\ 1 & 0.943847 & 2.200771 & 0.016673 \\ 1 & 0.359638 & 1.357725 & -1.426010 \\ 1 & -0.779914 & 2.313710 & -0.455257\end{array}$

Lowest Frequency $=-328.8554 \mathrm{~cm}^{-1}$

Energy $=-690.239803$ Hartrees

Zero Point Correction $=0.089443$ Hartrees 
(R)-Inversion Transition State $\mathbf{B}$ MP2/6-311+G*

\begin{tabular}{cc} 
& $\mathrm{X}$ \\
6 & 2.271007 \\
6 & 1.14344 \\
6 & 0.240823 \\
17 & -2.10365 \\
8 & 1.099426 \\
1 & 3.18733 \\
1 & 2.28923 \\
1 & -0.04317 \\
6 & -0.01608 \\
1 & -1.06733 \\
1 & 0.174409 \\
1 & 0.59111 \\
\multicolumn{2}{|c}{} \\
Frequency $=-375.7976 \mathrm{~cm}^{-1}$ \\
$=-690.230236$ Hartrees \\
Point Correction = 0.089459 Hartrees
\end{tabular}

$\begin{array}{cc}\mathrm{Y} & \mathrm{Z} \\ 0.321827 & -0.072845 \\ -0.560636 & -0.120322 \\ 0.440386 & 0.435109 \\ -0.343738 & -0.050906 \\ -1.792136 & -0.063629 \\ -0.043560 & 0.389849 \\ 1.295188 & -0.540731 \\ 0.254304 & 1.467089 \\ 1.825918 & -0.108813 \\ 2.059254 & 0.047551 \\ 1.865164 & -1.183614 \\ 2.585320 & 0.395522\end{array}$

Lowest Frequency $=-375.7976 \mathrm{~cm}^{-1}$

Energy $=-690.230236$ Hartrees

Zero Point Correction $=0.089459$ Hartrees

\section{$\underline{\text { Structure } 10}$}

Retention Ground State MP2/6-311+G*

$\begin{array}{cccc} & \mathrm{X} & \mathrm{Y} & \mathrm{Z} \\ 6 & 1.193656 & 0.352155 & 0.000006 \\ 8 & 2.150951 & -0.499881 & -0.000023 \\ 6 & 1.269474 & 1.730015 & 0.000013 \\ 1 & 2.254400 & 2.190264 & -0.000006 \\ 1 & 0.396947 & 2.367823 & 0.000017 \\ 6 & -0.183621 & -0.376035 & 0.000008 \\ 17 & -1.642839 & 0.737454 & -0.000023 \\ 6 & -0.305753 & -1.228108 & 1.258388 \\ 1 & -0.281523 & -0.589926 & 2.145838 \\ 1 & 0.550902 & -1.904651 & 1.291628 \\ 1 & -1.238829 & -1.802846 & 1.257619 \\ 6 & -0.305733 & -1.228152 & -1.258345 \\ 1 & 0.550923 & -1.904695 & -1.291549 \\ 1 & -0.281491 & -0.590000 & -2.145816 \\ 1 & -1.238808 & -1.802891 & -1.257571\end{array}$

Lowest Frequency $=58.9769 \mathrm{~cm}^{-1}$

Energy $=-729.465175$ Hartrees

Zero Point Correction $=0.119240$ Hartrees 
Retention Transition State MP2/6-311+G*

$\begin{array}{cccc} & \mathrm{X} & \mathrm{Y} & \mathrm{Z} \\ 6 & -1.446545 & -0.356005 & -0.052408 \\ 8 & -2.360744 & -0.025284 & -0.857527 \\ 6 & -1.113247 & -1.673122 & 0.363039 \\ 1 & -1.495711 & -2.509591 & -0.218504 \\ 1 & -0.349700 & -1.874600 & 1.100591 \\ 6 & -0.347410 & 0.516835 & 0.305377 \\ 17 & 2.186066 & -0.471858 & -0.467338 \\ 6 & 0.177932 & 0.626542 & 1.696160 \\ 1 & 0.090187 & -0.299479 & 2.265337 \\ 1 & 1.214485 & 0.960578 & 1.697640 \\ 1 & -0.446001 & 1.379278 & 2.206931 \\ 6 & -0.109520 & 1.668279 & -0.603653 \\ 1 & 0.853091 & 2.140792 & -0.415620 \\ 1 & -0.178508 & 1.348547 & -1.643711 \\ 1 & -0.932269 & 2.383163 & -0.438794\end{array}$

Lowest Frequency $=-216.9943 \mathrm{~cm}^{-1}$

Energy $=-729.416539$ Hartrees

Zero Point Correction $=0.117284$ Hartrees

Inversion Ground State MP2/6-311+G*

$\begin{array}{cc} & \mathrm{X} \\ 6 & 1.187161 \\ 8 & 1.374161 \\ 6 & 2.103697 \\ 1 & 3.106215 \\ 1 & 1.857757 \\ 6 & -0.232587 \\ 17 & -1.439845 \\ 6 & -0.478224 \\ 1 & -1.510266 \\ 1 & 0.202419 \\ 1 & -0.312514 \\ 6 & -0.572151 \\ 1 & -1.595016 \\ 1 & 0.131976 \\ 1 & -0.443867\end{array}$

$\mathrm{Y}$
-0.263393
-1.471247
0.610115
0.244409
1.618905
0.297190
-0.614915
1.772501
2.037776
2.378375
2.003244
-0.036157
0.267736
0.497450
-1.105904

$\mathrm{Z}$

$-0.070907$

0.280808

$-0.639658$

$-0.847970$

$-0.945534$

0.250043

$-0.794449$

$-0.034451$

0.219222

0.570895

$-1.088099$

1.695275

1.945158

2.344696

1.858995

Lowest Frequency $=62.9214 \mathrm{~cm}^{-1}$

Energy $=-729.462488$ Hartrees

Zero Point Correction $=0.119299$ Hartrees 
Inversion Transition State MP2/6-311+G*

$\begin{array}{cccc} & \mathrm{X} & \mathrm{Y} & \mathrm{Z} \\ 6 & 1.134783 & -0.624178 & -0.293496 \\ 8 & 1.123481 & -1.824449 & 0.004845 \\ 6 & 2.256301 & 0.220566 & -0.571446 \\ 1 & 3.232204 & -0.096517 & -0.203585 \\ 1 & 2.202314 & 1.121898 & -1.163526 \\ 6 & 0.290740 & 0.451488 & 0.208940 \\ 17 & -2.115697 & -0.422321 & -0.348221 \\ 6 & -0.008388 & 1.745633 & -0.508099 \\ 1 & -1.058076 & 1.982607 & -0.339657 \\ 1 & 0.604132 & 2.570071 & -0.121148 \\ 1 & 0.133936 & 1.652670 & -1.586051 \\ 6 & 0.003602 & 0.422188 & 1.687396 \\ 1 & -0.942301 & 0.923016 & 1.895585 \\ 1 & 0.809002 & 0.937748 & 2.229533 \\ 1 & -0.064430 & -0.610620 & 2.030068\end{array}$

Lowest Frequency $=-323.0363 \mathrm{~cm}^{-1}$

Energy $=-729.419631$ Hartrees

Zero Point Correction $=0.117585$ Hartrees

\section{$\underline{\text { Structure } 11}$}

Retention Ground State MP2/6-311+G*

$\begin{array}{cccc} & \mathrm{X} & \mathrm{Y} & \mathrm{Z} \\ 6 & -1.871714 & -0.059150 & 1.257351 \\ 6 & -0.386294 & -0.420845 & 1.262950 \\ 6 & 0.368057 & 0.003563 & 0.000006 \\ 6 & -0.386323 & -0.421201 & -1.262802 \\ 6 & -1.871744 & -0.059510 & -1.257272 \\ 6 & -2.561652 & -0.592552 & 0.000124 \\ 6 & 1.778492 & -0.663727 & 0.000081 \\ 6 & 2.924809 & 0.104366 & 0.000047 \\ 8 & 1.677500 & -1.941763 & 0.000063 \\ 17 & 0.407332 & 1.840537 & -0.000252 \\ 1 & 3.884466 & -0.406744 & 0.000036 \\ 1 & 2.911315 & 1.184915 & 0.000008 \\ 1 & 0.122939 & 0.012455 & -2.131632 \\ 1 & -0.259783 & -1.508588 & -1.320413 \\ 1 & -1.992809 & 1.030344 & -1.302446 \\ 1 & -1.992777 & 1.030717 & 1.302216 \\ 1 & -3.626260 & -0.319543 & 0.000098 \\ 1 & -2.351394 & -0.468639 & 2.157163 \\ 1 & -2.513920 & -1.691184 & 0.000281 \\ 1 & -0.259753 & -1.508214 & 1.320864 \\ 1 & 0.122990 & 0.013055 & 2.131646 \\ 1 & -2.351446 & -0.469256 & -2.156956\end{array}$

Lowest Frequency $=56.8907 \mathrm{~cm}^{-1}$

Energy $=-845.843760$ Hartrees

Zero Point Correction $=0.186245$ Hartrees 
Retention Transition State MP2/6-311+G*

$\begin{array}{cc} & X \\ 6 & -1.703952 \\ 6 & -0.218714 \\ 6 & 0.482616 \\ 6 & -0.292899 \\ 6 & -1.773039 \\ 6 & -2.421089 \\ 6 & 1.925062 \\ 6 & 2.472841 \\ 8 & 2.544849 \\ 17 & -0.166457 \\ 1 & 3.452727 \\ 1 & 1.883354 \\ 1 & -0.217786 \\ 1 & 0.205186 \\ 1 & -1.853444 \\ 1 & -1.797844 \\ 1 & -3.483190 \\ 1 & -2.159035 \\ 1 & -2.381373 \\ 1 & 0.346975 \\ 1 & -0.071052 \\ 1 & -2.278504\end{array}$

$\mathrm{Y}$
-0.685019
-1.019670
-0.503343
-0.420675
-0.070463
-0.908845
-0.615581
0.476259
-1.384121
2.263841
0.848508
1.078598
-1.442044
0.227032
0.990581
0.364809
-0.647874
-1.300248
-1.976006
-0.713336
-2.116246
-0.242083

$\mathrm{Z}$

$-1.160414$

$-1.059216$

0.147519

1.422895

1.273193

0.171149

0.125859

0.852517

$-0.662184$

$-0.521176$

0.560036

1.528300

1.845779

2.151173

1.025027

$-1.451000$

0.076254

$-1.948448$

0.442660

$-1.944938$

$-1.001990$

2.233605

Lowest Frequency $=-173.1116 \mathrm{~cm}^{-1}$

Energy $=-845.795513$ Hartrees

Zero Point Correction $=0.184069$ Hartrees 
Inversion Ground State MP2/6-311+G*

$\begin{array}{cccc} & \mathrm{X} & \mathrm{Y} & \mathrm{Z} \\ 6 & 1.924336 & 0.948739 & 0.772520 \\ 6 & 0.431536 & 1.231174 & 0.581411 \\ 6 & -0.351854 & 0.052209 & 0.005525 \\ 6 & 0.347397 & -0.505949 & -1.232827 \\ 6 & 1.838186 & -0.781109 & -1.045983 \\ 6 & 2.568777 & 0.457642 & -0.524687 \\ 6 & -1.849683 & 0.302434 & -0.355468 \\ 6 & -2.384500 & 1.543909 & -0.042069 \\ 8 & -2.415962 & -0.662873 & -0.960400 \\ 17 & -0.335827 & -1.266305 & 1.293219 \\ 1 & -3.423154 & 1.739811 & -0.297882 \\ 1 & -1.849388 & 2.298755 & 0.520340 \\ 1 & -0.205720 & -1.391849 & -1.553099 \\ 1 & 0.203780 & 0.250366 & -2.019994 \\ 1 & 1.971394 & -1.607333 & -0.336461 \\ 1 & 2.060113 & 0.192255 & 1.554811 \\ 1 & 3.634640 & 0.241983 & -0.366250 \\ 1 & 2.423724 & 1.862206 & 1.124138 \\ 1 & 2.516041 & 1.255145 & -1.280936 \\ 1 & 0.308715 & 2.062708 & -0.124757 \\ 1 & -0.023181 & 1.540407 & 1.527610 \\ 1 & 2.274629 & -1.108579 & -1.999579\end{array}$

Lowest Frequency $=67.7595 \mathrm{~cm}^{-1}$

Energy $=-845.840777$ Hartrees

Zero Point Correction $=0.186242$ Hartrees 
Inversion Transition State MP2/6-311+G*

$\begin{array}{cccc} & \mathrm{X} & \mathrm{Y} & \mathrm{Z} \\ 6 & 1.848921 & -0.395217 & -1.089288 \\ 6 & 0.377737 & -0.808331 & -1.227822 \\ 6 & -0.481106 & -0.444267 & -0.025947 \\ 6 & 0.150094 & -0.580725 & 1.341545 \\ 6 & 1.631185 & -0.195869 & 1.400080 \\ 6 & 2.420018 & -0.833196 & 0.258108 \\ 6 & -1.924764 & -0.259890 & -0.012695 \\ 6 & -2.214701 & -1.508988 & -0.650479 \\ 8 & -2.618080 & 0.368496 & 0.794624 \\ 17 & 0.056051 & 2.196525 & -0.353785 \\ 1 & -2.966987 & -2.154129 & -0.195419 \\ 1 & -1.766037 & -1.818790 & -1.583079 \\ 1 & -0.439036 & 0.018505 & 2.041419 \\ 1 & 0.048515 & -1.638103 & 1.639898 \\ 1 & 1.706736 & 0.892081 & 1.330615 \\ 1 & 1.929162 & 0.687827 & -1.190152 \\ 1 & 3.478723 & -0.549363 & 0.326638 \\ 1 & 2.411061 & -0.861743 & -1.910927 \\ 1 & 2.380876 & -1.931427 & 0.343706 \\ 1 & 0.377966 & -1.901377 & -1.347208 \\ 1 & -0.053244 & -0.366129 & -2.130930 \\ 1 & 2.039737 & -0.507344 & 2.371777\end{array}$

Lowest Frequency $=-282.0013 \mathrm{~cm}^{-1}$

Energy $=-845.794310$ Hartrees

Zero Point Correction $=0.184663$ Hartrees 


\section{$\underline{\text { Structure } \mathbf{1 2}}$}

Retention Ground State MP2/6-311+G*

$\begin{array}{cccc} & \mathrm{X} & \mathrm{Y} & \mathrm{Z} \\ 6 & -0.019853 & 1.233516 & 0.135507 \\ 8 & 0.729867 & 2.264423 & 0.203914 \\ 6 & -1.329274 & 1.157985 & -0.326808 \\ 6 & 0.595960 & -0.069063 & 0.666859 \\ 6 & -0.387613 & -1.198301 & 0.932041 \\ 6 & -1.351703 & -1.354542 & -0.236943 \\ 6 & -2.185744 & -0.082158 & -0.370036 \\ 1 & -1.782846 & 2.102210 & -0.634466 \\ 1 & 1.166708 & 0.182956 & 1.564895 \\ 17 & 1.890249 & -0.676261 & -0.483902 \\ 1 & 0.152194 & -2.129811 & 1.145324 \\ 1 & -0.958532 & -0.932088 & 1.834571 \\ 1 & -1.997768 & -2.231866 & -0.088527 \\ 1 & -0.775765 & -1.517122 & -1.155533 \\ 1 & -2.948820 & -0.091262 & 0.434915 \\ 1 & -2.758979 & -0.126597 & -1.309868\end{array}$

Lowest Frequency $=88.0127 \mathrm{~cm}^{-1}$

Energy $=-767.469947$ Hartrees

Zero Point Correction $=0.129216$ Hartrees

Retention Transition State MP2/6-311+G*

$\begin{array}{cccc} & \mathrm{X} & \mathrm{Y} & \mathrm{Z} \\ 6 & 1.183712 & -1.010523 & 0.104908 \\ 8 & 1.635879 & -2.179782 & 0.114866 \\ 6 & 1.470518 & -0.000092 & -0.884656 \\ 6 & 0.058234 & -0.539539 & 0.863798 \\ 6 & -0.106831 & 0.885524 & 1.278044 \\ 6 & 0.080685 & 1.834364 & 0.094100 \\ 6 & 1.378806 & 1.474511 & -0.634071 \\ 1 & 1.845183 & -0.364240 & -1.844102 \\ 1 & -0.586955 & -1.292516 & 1.307065 \\ 17 & -2.430370 & -0.428977 & -0.431680 \\ 1 & -1.095431 & 1.020193 & 1.721330 \\ 1 & 0.658479 & 1.118694 & 2.044610 \\ 1 & 0.096900 & 2.882425 & 0.425934 \\ 1 & -0.772807 & 1.681959 & -0.572306 \\ 1 & 2.236343 & 1.798736 & -0.013835 \\ 1 & 1.456794 & 2.020151 & -1.581805\end{array}$

Lowest Frequency $=-222.7632 \mathrm{~cm}^{-1}$

Energy $=-767.425472$ Hartrees

Zero Point Correction $=0.126778$ Hartrees 
Inversion Ground State

MP2/6-311+G*

$\begin{array}{cccc} & \mathrm{X} & \mathrm{Y} & \mathrm{Z} \\ 6 & 0.000583 & 1.138937 & 0.105674 \\ 8 & 0.815286 & 2.098087 & 0.291675 \\ 6 & -1.336965 & 1.223324 & -0.274047 \\ 6 & 0.516571 & -0.274795 & 0.455964 \\ 6 & -0.374489 & -1.433147 & 0.030896 \\ 6 & -1.827738 & -1.139607 & 0.386130 \\ 6 & -2.291847 & 0.067063 & -0.426970 \\ 1 & -1.715690 & 2.231423 & -0.451150 \\ 1 & 0.707784 & -0.301914 & 1.535243 \\ 17 & 2.162260 & -0.555592 & -0.269345 \\ 1 & -0.293569 & -1.557200 & -1.056779 \\ 1 & -0.017258 & -2.365586 & 0.488685 \\ 1 & -2.452727 & -2.022381 & 0.181970 \\ 1 & -1.912371 & -0.911684 & 1.457539 \\ 1 & -2.413271 & -0.265403 & -1.477763 \\ 1 & -3.300302 & 0.362461 & -0.098157\end{array}$

Lowest Frequency $=79.1368 \mathrm{~cm}^{-1}$

Energy $=-767.467588$ Hartrees

Zero Point Correction $=0.129077$ Hartrees

Inversion Transition State

MP2/6-311+G*

$\begin{array}{cccc} & \mathrm{X} & \mathrm{Y} & \mathrm{Z} \\ 6 & -0.248136 & 1.284984 & -0.046256 \\ 8 & 0.281428 & 2.401095 & -0.060752 \\ 6 & -1.645025 & 0.948726 & 0.099527 \\ 6 & 0.217954 & 0.029463 & 0.520325 \\ 6 & -0.216048 & -1.329627 & 0.027529 \\ 6 & -1.725766 & -1.519818 & 0.178682 \\ 6 & -2.345738 & -0.269706 & -0.445853 \\ 1 & -2.233679 & 1.698106 & 0.634926 \\ 1 & 0.668858 & 0.117059 & 1.504948 \\ 17 & 2.611340 & -0.461057 & -0.138499 \\ 1 & 0.060249 & -1.423450 & -1.028843 \\ 1 & 0.361846 & -2.086084 & 0.561495 \\ 1 & -2.067438 & -2.449506 & -0.302958 \\ 1 & -1.996813 & -1.562979 & 1.241575 \\ 1 & -2.241092 & -0.332521 & -1.540457 \\ 1 & -3.419589 & -0.195547 & -0.233906\end{array}$

Lowest Frequency $=-269.2217 \mathrm{~cm}^{-1}$

Energy $=-767.430380$ Hartrees

Zero Point Correction $=0.127168$ Hartrees 


\section{$\underline{\text { Structure } 13}$}

Retention Ground State MP2/6-311+G*

$\begin{array}{cccc} & \mathrm{X} & \mathrm{Y} & \mathrm{Z} \\ 6 & -1.989424 & 0.852401 & 0.029372 \\ 6 & -0.763058 & 1.646481 & -0.343476 \\ 6 & 0.551840 & 1.315933 & -0.035372 \\ 6 & 0.764389 & 0.005072 & 0.738283 \\ 6 & -0.493141 & -0.688694 & 1.250105 \\ 6 & -1.674631 & -0.626272 & 0.280946 \\ 8 & 1.607898 & 1.985223 & -0.287668 \\ 6 & -1.452231 & -1.390302 & -1.026331 \\ 17 & 1.764002 & -1.168818 & -0.258638 \\ 1 & -0.909728 & 2.614018 & -0.827519 \\ 1 & 1.438988 & 0.229243 & 1.569975 \\ 1 & -0.265343 & -1.724745 & 1.535021 \\ 1 & -0.792570 & -0.161491 & 2.169155 \\ 1 & -2.537547 & -1.083800 & 0.793099 \\ 1 & -2.484464 & 1.247093 & 0.939948 \\ 1 & -2.751113 & 0.923185 & -0.764783 \\ 1 & -2.390934 & -1.428368 & -1.593028 \\ 1 & -0.700455 & -0.896807 & -1.643490 \\ 1 & -1.120518 & -2.417921 & -0.841358\end{array}$

Lowest Frequency $=78.8621 \mathrm{~cm}^{-1}$

Energy $=-806.651646$ Hartrees

Zero Point Correction $=0.157473$ Hartrees

Retention Transition State MP2/6-311+G*

$\begin{array}{cccc} & \mathrm{X} & \mathrm{Y} & \mathrm{Z} \\ 6 & -1.858157 & -1.106318 & -0.258787 \\ 6 & -1.662465 & 0.274182 & -0.799300 \\ 6 & -1.098877 & 1.350060 & -0.015084 \\ 6 & -0.037852 & 0.807525 & 0.787330 \\ 6 & -0.051883 & -0.568662 & 1.361563 \\ 6 & -0.582764 & -1.650460 & 0.404563 \\ 8 & -1.340660 & 2.569326 & -0.170745 \\ 6 & 0.445129 & -2.077478 & -0.639939 \\ 17 & 2.619353 & 0.503367 & -0.280462 \\ 1 & -2.084664 & 0.547983 & -1.769129 \\ 1 & 0.719782 & 1.500805 & 1.139004 \\ 1 & 0.952735 & -0.817273 & 1.713702 \\ 1 & -0.725685 & -0.549656 & 2.242851 \\ 1 & -0.859248 & -2.530606 & 1.006808 \\ 1 & -2.662460 & -1.089204 & 0.501415 \\ 1 & -2.194166 & -1.784921 & -1.052835 \\ 1 & 0.014565 & -2.852220 & -1.287241 \\ 1 & 0.775342 & -1.231054 & -1.243363 \\ 1 & 1.341285 & -2.478801 & -0.159474\end{array}$

Lowest Frequency $=-214.3667 \mathrm{~cm}^{-1}$

Energy $=-806.607014$ Hartrees

Zero Point Correction $=0.154773$ Hartrees 
Inversion Ground State MP2/6-311+G*

$\begin{array}{cccc} & \mathrm{X} & \mathrm{Y} & \mathrm{Z} \\ 6 & -1.690653 & 1.102004 & -0.444248 \\ 6 & -0.356804 & 1.782827 & -0.273167 \\ 6 & 0.830731 & 1.168954 & 0.116290 \\ 6 & 0.732294 & -0.336308 & 0.449806 \\ 6 & -0.541908 & -1.032559 & -0.002796 \\ 6 & -1.770196 & -0.200952 & 0.350552 \\ 8 & 1.959778 & 1.719090 & 0.319357 \\ 6 & -3.061184 & -0.973481 & 0.082626 \\ 17 & 2.137195 & -1.246671 & -0.264120 \\ 1 & -0.303218 & 2.859826 & -0.440701 \\ 1 & 0.878937 & -0.451594 & 1.530311 \\ 1 & -0.508694 & -1.157750 & -1.094240 \\ 1 & -0.595951 & -2.040105 & 0.434788 \\ 1 & -1.725121 & 0.057603 & 1.420059 \\ 1 & -1.926858 & 0.855625 & -1.500143 \\ 1 & -2.505022 & 1.767424 & -0.113090 \\ 1 & -3.112064 & -1.279949 & -0.968877 \\ 1 & -3.941613 & -0.355340 & 0.293655 \\ 1 & -3.124616 & -1.877958 & 0.699055\end{array}$

Lowest Frequency $=73.2702 \mathrm{~cm}^{-1}$

Energy $=-806.653430$ Hartrees

Zero Point Correction $=0.157155$ Hartrees

Inversion Transition State

MP2/6-311+G*

$\begin{array}{cccc} & \mathrm{X} & \mathrm{Y} & \mathrm{Z} \\ 6 & -1.900315 & 0.917056 & -0.346146 \\ 6 & -0.729714 & 1.750034 & 0.105933 \\ 6 & 0.669654 & 1.452192 & -0.089232 \\ 6 & 0.584900 & 0.145091 & 0.541334 \\ 6 & -0.408990 & -0.916353 & 0.143345 \\ 6 & -1.853116 & -0.449403 & 0.341873 \\ 8 & 1.621429 & 2.234770 & -0.182332 \\ 6 & -2.878854 & -1.445721 & -0.190473 \\ 17 & 2.504352 & -1.363894 & -0.138396 \\ 1 & -0.922198 & 2.699625 & 0.611701 \\ 1 & 1.076543 & 0.075076 & 1.507778 \\ 1 & -0.256305 & -1.165163 & -0.914673 \\ 1 & -0.185771 & -1.826825 & 0.704534 \\ 1 & -2.022332 & -0.284307 & 1.416882 \\ 1 & -1.879771 & 0.753896 & -1.436495 \\ 1 & -2.837200 & 1.445180 & -0.123695 \\ 1 & -2.723569 & -1.618788 & -1.261282 \\ 1 & -3.901742 & -1.076609 & -0.052312 \\ 1 & -2.794481 & -2.411430 & 0.319146\end{array}$

Lowest Frequency $=-257.8075 \mathrm{~cm}^{-1}$

Energy $=-806.616149$ Hartrees

Zero Point Correction $=0.155176$ Hartrees 


\section{$\underline{\text { Structure } 14}$}

Retention Ground State MP2/6-311+G*

$\begin{array}{cccc} & \mathrm{X} & \mathrm{Y} & \mathrm{Z} \\ 6 & -1.427315 & 1.324001 & -0.357161 \\ 6 & 0.000334 & 1.807368 & -0.328366 \\ 6 & 1.096939 & 1.083898 & 0.126590 \\ 6 & 0.808185 & -0.324384 & 0.666440 \\ 6 & -0.654771 & -0.631875 & 0.939604 \\ 6 & -1.537420 & -0.193714 & -0.222184 \\ 8 & 2.316411 & 1.456819 & 0.185043 \\ 6 & -2.981600 & -0.655394 & -0.039009 \\ 17 & 1.466158 & -1.597568 & -0.480362 \\ 1 & 0.197459 & 2.833984 & -0.642926 \\ 1 & 1.420971 & -0.463321 & 1.561446 \\ 1 & -0.785795 & -1.699915 & 1.162312 \\ 1 & -0.952419 & -0.067459 & 1.837975 \\ 1 & -1.134726 & -0.645287 & -1.139277 \\ 1 & -2.039480 & 1.773944 & 0.452547 \\ 1 & -1.924787 & 1.625414 & -1.294550 \\ 1 & -3.617415 & -0.295967 & -0.856554 \\ 1 & -3.050811 & -1.749247 & -0.010743 \\ 1 & -3.395086 & -0.267439 & 0.900093\end{array}$

Lowest Frequency $=79.2274 \mathrm{~cm}^{-1}$

Energy $=-806.655998$ Hartrees

Zero Point Correction $=0.157319$ Hartrees

Retention Transition State MP2/6-311+G*

$\begin{array}{cccc} & \mathrm{X} & \mathrm{Y} & \mathrm{Z} \\ 6 & 0.988656 & -1.441344 & -0.752269 \\ 6 & -0.500991 & -1.485588 & -0.892194 \\ 6 & -1.422692 & -1.123497 & 0.158758 \\ 6 & -0.844509 & -0.020046 & 0.874388 \\ 6 & 0.615575 & 0.080352 & 1.161136 \\ 6 & 1.456491 & -0.135848 & -0.099889 \\ 8 & -2.608934 & -1.517959 & 0.260433 \\ 6 & 2.952802 & -0.129147 & 0.186347 \\ 17 & -0.697766 & 2.475235 & -0.418956 \\ 1 & -0.949497 & -1.877383 & -1.808175 \\ 1 & -1.528212 & 0.656436 & 1.379520 \\ 1 & 0.836466 & 1.057509 & 1.597823 \\ 1 & 0.891219 & -0.705823 & 1.893651 \\ 1 & 1.199724 & 0.695887 & -0.765943 \\ 1 & 1.332796 & -2.284279 & -0.119854 \\ 1 & 1.466724 & -1.572343 & -1.731748 \\ 1 & 3.528803 & -0.261656 & -0.736475 \\ 1 & 3.253438 & 0.822926 & 0.635555 \\ 1 & 3.230039 & -0.935892 & 0.876784\end{array}$

Lowest Frequency $=-211.3392 \mathrm{~cm}^{-1}$

Energy $=-806.612266$ Hartrees

Zero Point Correction $=0.154871$ Hartrees 
Inversion Ground State MP2/6-311+G*

$\begin{array}{cccc} & \mathrm{X} & \mathrm{Y} & \mathrm{Z} \\ 6 & -1.863788 & 0.607518 & -0.929786 \\ 6 & -0.801945 & 1.552024 & -0.424950 \\ 6 & 0.418259 & 1.188949 & 0.137791 \\ 6 & 0.646872 & -0.325419 & 0.323697 \\ 6 & -0.316114 & -1.243403 & -0.417343 \\ 6 & -1.763301 & -0.774058 & -0.271536 \\ 8 & 1.313593 & 1.957798 & 0.615500 \\ 6 & -2.225287 & -0.760296 & 1.187465 \\ 17 & 2.333102 & -0.791092 & -0.177896 \\ 1 & -1.002920 & 2.623801 & -0.469639 \\ 1 & 0.643554 & -0.530865 & 1.400327 \\ 1 & -0.050801 & -1.236992 & -1.482198 \\ 1 & -0.193930 & -2.276955 & -0.061757 \\ 1 & -2.403083 & -1.481642 & -0.825035 \\ 1 & -1.822085 & 0.452269 & -2.026215 \\ 1 & -2.865416 & 1.025984 & -0.735965 \\ 1 & -2.044620 & -1.728753 & 1.670513 \\ 1 & -3.299399 & -0.548260 & 1.247407 \\ 1 & -1.700957 & 0.015709 & 1.750767\end{array}$

Lowest Frequency $=71.4241 \mathrm{~cm}^{-1}$

Energy $=-806.652178$ Hartrees

Zero Point Correction $=0.157359$ Hartrees

Inversion Transition State

MP2/6-311+G*

$\begin{array}{cccc} & \mathrm{X} & \mathrm{Y} & \mathrm{Z} \\ 6 & -1.958719 & 0.554356 & -0.842679 \\ 6 & -1.076271 & 1.451879 & -0.012637 \\ 6 & 0.366565 & 1.441056 & 0.038908 \\ 6 & 0.438740 & 0.034816 & 0.404091 \\ 6 & -0.234037 & -1.070902 & -0.374005 \\ 6 & -1.758036 & -0.904774 & -0.414015 \\ 8 & 1.141353 & 2.372090 & 0.283193 \\ 6 & -2.413912 & -1.196902 & 0.934048 \\ 17 & 2.699939 & -0.937342 & -0.081414 \\ 1 & -1.540669 & 2.229857 & 0.598849 \\ 1 & 0.741555 & -0.147223 & 1.431785 \\ 1 & 0.159501 & -1.064127 & -1.396652 \\ 1 & 0.066472 & -2.027287 & 0.061070 \\ 1 & -2.180025 & -1.589147 & -1.168778 \\ 1 & -1.718049 & 0.641991 & -1.913621 \\ 1 & -3.005818 & 0.866234 & -0.728872 \\ 1 & -2.199354 & -2.220618 & 1.259452 \\ 1 & -3.502633 & -1.079375 & 0.875787 \\ 1 & -2.036757 & -0.509377 & 1.697195\end{array}$

Lowest Frequency $=-273.2232 \mathrm{~cm}^{-1}$

Energy $=-806.615605$ Hartrees

Zero Point Correction $=0.155371$ Hartrees 